\title{
Robust Predictions for DSGE Models with Incomplete Information*
}

\author{
Ryan Chahrour \\ Boston College
}

\author{
Robert Ulbricht \\ Toulouse School of Economics
}

March 14, 2019

\begin{abstract}
We study the quantitative potential of DSGE models with incomplete information. In contrast to existing literature, we offer predictions that are robust across all possible private information structures that agents may have. Our approach maps a DSGE model with information-frictions into a parallel economy where deviations from fullinformation are captured by time-varying wedges. We derive exact conditions that ensure the consistency of these wedges with some information structure. We apply our approach to an otherwise frictionless business cycle model where firms and households have incomplete information. We show how assumptions about information interact with the presence of idiosyncratic shocks to shape the potential for confidence-driven fluctuations. For a realistic calibration, we find that correlated confidence regarding idiosyncratic shocks (aka "sentiment shocks") can account for up to 51 percent of U.S. business cycle fluctuations.
\end{abstract}

Keywords: Business cycles, DSGE models, incomplete-information, information-robust predictions.

JEL Classification: E32, D84.

\footnotetext{
*This paper previously circulated under the title "Information-driven Business Cycles: A Primal Approach". We would like to thank our discussants Guillaume Chevillon, Stéphane Dupraz, Alexandre Kohlhas, Jianjun Miao and Anna Orlik for many valuable comments. Ulbricht gratefully acknowledges financial support from the European Union's Horizon 2020 Program under grant agreement No. 649396. Email addresses: ryan.chahrour@bc.edu,robert.ulbricht@tse-fr.eu.
} 


\section{Introduction}

What are the sources of aggregate fluctuations? A popular view is that business cycles are caused by shocks to the confidence of consumers and firms. The view has been formalized in recent business cycle models based on incomplete information (e.g., Lorenzoni, 2009; Angeletos and La'O, 2013; Benhabib, Wang and Wen, 2015). Yet, few of these models have been investigated quantitatively. At least in part, this is because the private information structures governing people's beliefs are hard to observe in the data or - as argued by Sims (2003) and Woodford (2003) - may have no observable counterpart.

In this paper, we quantify the potential of confidence-driven business cycles using a novel approach that bypasses the challenge of postulating ad-hoc information structures. The approach takes the economic environment (technology, preferences, markets structure) as given, but does not require a detailed specification of the information structure that governs agents' beliefs. Instead, we provide an "information-robust" characterization of all equilibria that are possible within a given economic environment.

To do this, we map DSGE models with incomplete information into a "primal" economy in which deviations from full information are summarized by wedges in agents' equilibrium expectations. We then develop necessary and sufficient conditions for the existence of an information structure that is consistent with the expectation errors captured by these wedges. Subject to these conditions, the primal economy is isomorphic to the incomplete-information economy. Exploiting this equivalence, we derive a complete characterization of all equilibria within a given economic environment independently from the information structure. The characterization is quite general and applicable to a large class of linear rational expectation models.

For a concrete application, we use our approach to ask: Under what conditions can changes in confidence generate sizable fluctuations in aggregate economic activity? We examine this question in the context of an otherwise frictionless business cycle model driven by shocks to aggregate productivity, along with shocks to local productivities and demand. We allow for a general form of imperfect information, in which households and firms have access to an arbitrary set of signals governing their beliefs about their own idiosyncratic shocks, the aggregate state of the economy, what other agents believe, and so on. This includes the possibility to learn from endogenous signals (e.g., Amador and Weill, 2010) along with many others.

Whether the model generates aggregate fluctuations beyond those induced by aggregate productivity shocks depends on its ability to generate expectation errors that are correlated in the cross-section. There are two potential sources of such correlation. First, agents can be 
jointly optimistic or pessimistic regarding the aggregate state of productivity, as in Lorenzoni (2009) or Angeletos and La'O (2010). Second, agents can be jointly optimistic or pessimistic about their own idiosyncratic conditions, as in Angeletos and La'O (2013) or Benhabib, Wang and Wen (2015), possibly accentuated by strategic uncertainty and higher-order beliefs. Both channels are disciplined by the properties of the fundamental shocks to productivity and demand. Our approach allows us to provide a general characterization of these restrictions that does not hinge on a specific structural assumption about people's information.

As a first result, we establish a novel theoretical benchmark for the case in which idiosyncratic shocks are unrestricted by data. In this case, it has been previously demonstrated that one can generate arbitrary macroeconomic volatility through confidence-driven fluctuations if the idiosyncratic shocks are sufficiently volatile (Angeletos and La'O, 2013; Benhabib, Wang and Wen, 2015). We go further, showing that confidence-driven fluctuations can in principle generate any dynamic process for output and inflation, potentially bypassing all cross-equation restrictions that obtain under full information, provided that agents do not perfectly observe demand for their local goods when making production choices. If, on the other hand, we impose some minimal requirements on the information of agents, we obtain non-trivial cross-equation restrictions. For example, when local demand is perfectly observed by firms, inflation must be procyclical regardless of what else firms might observe.

For our quantitative analysis, we use existing micro-data estimates (Foster, Haltiwanger and Syverson, 2008) to calibrate the processes for idiosyncratic productivity and demand. We then compute global upper bounds on confidence-induced output fluctuations, their persistence, and the contemporaneous correlation with inflation (Figure 1 in Section 5). For an empirically plausible calibration, the volatility-frontier for confidence-induced output fluctuations is hump-shaped in aggregate persistence and is decreasing in the contemporaneous correlation with inflation. For an aggregate persistence and inflation-cyclicality consistent with U.S. data, the maximal one-step-ahead volatility of confidence-induced fluctuations is .011 ( $\approx 90$ percent of the empirical one-step-ahead volatility of the U.S. output gap). We demonstrate that the ability to generate sizable macro-volatility through confidence-fluctuations hinges on the volatility of idiosyncratic demand shocks, whereas shocks to productivity play a somewhat dispensable role.

Finally, we explore the degree to which confidence-driven fluctuations are consistent with U.S. business cycle data. To this end, we estimate a prototype wedge-economy similar to the one in Chari, Kehoe and McGrattan (2007), which captures the U.S. business cycle by construction. Our theoretical results permit us to partition the estimated wedges into an informational component, which can be microfounded through incomplete information, and 
a non-informational residual. We find that, in principle, confidence-fluctuations can account for a large portion of the U.S. business cycle that remains unexplained after conditioning on productivity shocks. The ability of the model to generate sizable aggregate confidencefluctuations hinges, however, crucially on the presumption that firms do not know their idiosyncratic product demands while making their production plans: If local demand is perfectly observed, at most 3 percent of observed output fluctuations can be accounted for by any type of confidence. By contrast, if local demand is not observed but aggregate productivity is, up to 51 percent of output fluctuations can be explained by correlated confidence regarding local conditions.

The methodology developed in this paper is closely related to Bergemann and Morris (2013, 2016) and Bergemann, Heumann and Morris (2014). These papers demonstrate the equivalence between Bayes equilibria in games with incomplete information and Bayes correlated equilibria. The approach developed in this paper is similar in that it also demonstrates the equivalence between a class of incomplete-information models with another class of full-information models. It is more general, however, as it is not limited to static game environments, but equally applies to dynamic market economies, which is crucial for an application to business cycles. Closely related to our application to dynamic macroeconomic models, Passadore and Xandri (2018) develop robust predictions in dynamic policy games with an application to sovereign debt.

On the applied side, our analysis relates to a recent literature on confidence-driven business cycles. While the literature is mostly theoretical, there are now a few studies with a quantitative focus. In particular, Huo and Takayama (2015a) quantify a version of Angeletos and La'O (2013), and Blanchard, L'Huillier and Lorenzoni (2013) estimate a version of Lorenzoni (2009). ${ }^{1}$ Our approach is distinguished by our general formulation of incomplete information that does not require an ex-ante stand on which agents are affected by information-frictions, how information is shared in the cross-section of agents, or any other parametric properties of the information structure.

The objective of this paper is also closely related to Angeletos, Collard and Dellas (2017). Departing from the assumption of rational expectations, those authors develop a tractable framework in which agents' expectations regarding the beliefs of other agents are subject to reduced-form "confidence shocks". They show that confidence shocks can account for a significant portion of the U.S. business cycle, but abstract from the question whether those

\footnotetext{
${ }^{1}$ See also Melosi $(2014,2016)$ for an estimation of a variant of Woodford (2003), and Maćkowiak and Wiederholt (2015) for plausible calibration of a particular DSGE model with rational inattention. In these works, incomplete information alternates the propagation of fundamental shocks (productivity, monetary), but there are no confidence-driven fluctuations.
} 
shocks can by microfounded by some information structure. Our approach is complimentary in that we characterize the restrictions on confidence-driven fluctuations imposed by rational expectations.

Our approach is also useful for reducing the computational burden of solving (and estimating) business cycle models with incomplete information. While the incomplete-information version of our economy is hard to solve, the corresponding primal economy permits a simple aggregate representation, in which aggregate wedges capture the average deviations from incomplete-information in the cross-section of agents. Conditional on these wedges, which are constrained by the theoretical restrictions characterized by our approach, the primal economy can be solved using standard tools developed for full-information models. In this ability to reduce the computational burden of solving (and estimating) incomplete information models, our paper also relates to Rondina and Walker (2014), Acharya (2013), Huo and Takayama (2015b) and Acharya, Benhabib and Huo (2017), who use frequency-domain techniques to obtain analytical solutions in certain models, and Nimark (2009) who explores the asymptotic accuracy of a finite-state approximation approach to a class of dispersed information models.

The rest of the paper is organized as follows. Section 2 sets up the model economy. Section 3 develops the information-robust characterization, and Section 4 applies it to derive predictions about aggregate fluctuations. Section 5 calibrates the model and provides the quantitative analysis. Section 6 concludes. A general statement and proof of our main representation result is contained in Appendix A.

\section{The Model Economy}

\subsection{Economic Environment}

The model is a standard RBC economy without capital, augmented with imperfect information. Households and firms are located on a continuum of islands, indexed by $i \in[0,1]$. On each island, a representative household interacts with a representative firm in a local labor market. Firms use the labor provided by households to produce differentiated intermediate goods, which are aggregated by a competitive final goods sector located on the mainland. There are no subperiods; all markets at date $t$ operate simultaneously. 
Households Preferences on island $i$ are given by

$$
\mathbb{E}\left\{\sum_{\tau=0}^{\infty} \beta^{\tau} U\left(C_{i, t+\tau}, N_{i, t+\tau}\right) \mid \mathcal{I}_{i, t}\right\}
$$

where $\beta \in(0,1)$ is the discount factor, $N_{i, t}$ is hours worked, $C_{i, t}$ is final good consumption, and $\mathcal{I}_{i, t}$ is the set of information available in island $i$ at time $t$. The utility flow $U$ is given by

$$
U(C, N)=\log C-\frac{1}{1+\zeta} N^{1+\zeta}
$$

where $\zeta \geq 0$ is the inverse of the Frisch elasticity of labor supply. The household's budget constraint is

$$
P_{t} C_{i, t}+Q_{t} B_{i, t} \leq W_{i, t} N_{i, t}+B_{i, t-1}+D_{i, t}
$$

where $P_{t}$ is the price of the final good, $Q_{t}$ is the nominal price of a riskless one-period bond, $B_{i, t}$ are local bond holdings, $W_{i, t}$ are local wage rates, and $D_{i, t}$ are profits of the local firm. ${ }^{2}$ Bonds are in zero net supply, so market clearing requires $\int_{0}^{1} B_{i, t} \mathrm{~d} i=0$. No other financial assets can be traded across islands, which implies that households are exposed to idiosyncratic income risk.

Intermediate-goods producers Each good $i$ is produced by a monopolistically competitive firm with access to a linear production technology,

$$
Y_{i, t}=A_{i, t} N_{i, t}
$$

Firms choose $N_{i, t}$ to maximize expected profits, $\mathbb{E}\left[P_{i, t} Y_{i, t}-W_{i, t} N_{i, t} \mid \mathcal{I}_{i, t}\right]$, subject to an inverse demand curve specified below. The wage rate $W_{i, t}$ is determined competitively. ${ }^{3}$ The productivity $A_{i, t}$ consists of an aggregate and an island-specific component,

$$
\log A_{i, t}=\log A_{t}+\Delta a_{i, t}
$$

\footnotetext{
${ }^{2}$ Following Maćkowiak and Wiederholt (2015), we assume that bond positions adjust to clear the budget constraint independently of the information available to households.

${ }^{3}$ Formally, firm $i$ is representative of a continuum of firms, $j \in[0,1]$, competing in the local labor market. Each of these firms produces a separate variety $(i, j)$ that is aggregated to $Y_{i, t}$ using the technology $Y_{i, t}=$ $\left(\int_{0}^{1} Y_{i j, t}^{1-1 / \eta} \mathrm{d} j\right)^{\eta /(\eta-1)}$ where $\eta$ matches the elasticity of substitution across "island-varieties" specified in the final good technology below. Clearly, the setting collapses to the one in the main text where $Y_{i, t}$ is produced by a representative firm $i$ that is competitive in the local labor market and faces isoelastic demand from the final good sector with elasticity $-\eta$.
} 
where the aggregate component follows a random walk process

$$
\log A_{t}=\log A_{t-1}+\epsilon_{t}
$$

The innovation $\epsilon_{t}$ is i.i.d. across time with zero mean and constant variance. The islandspecific component $\Delta a_{i, t}$ follows a time-invariant, stationary random process that is i.i.d. across islands and is normalized so that $\int_{0}^{1} \Delta a_{i, t} \mathrm{~d} i=0$.

Final-good sector A competitive final-goods sector aggregates intermediate input goods $i \in[0,1]$, using the technology

$$
Y_{t}=\left(\int_{0}^{1} Z_{i, t} Y_{i, t}^{\frac{\eta-1}{\eta}} \mathrm{d} i\right)^{\frac{\eta}{\eta-1}}
$$

where $\eta>1$ is the elasticity of substitution among input varieties, $Y_{i, t}$ denotes the input of intermediate good $i$ at time $t$, and $Z_{i, t}$ is an island-specific demand shifter following a time-invariant, stationary process that is i.i.d. across islands and satisfies $\int_{0}^{1} \log \left(Z_{i, t}\right) \mathrm{d} i=0$. Profit maximization yields the inverse input demands, given by

$$
P_{i, t}=\left(\frac{Y_{i, t}}{Y_{t}}\right)^{-1 / \eta} Z_{i, t} P_{t}
$$

where the aggregate price index $P_{t}$ is defined by

$$
P_{t}=\left(\int_{0}^{1} Z_{i, t}^{\eta} P_{i, t}^{1-\eta} \mathrm{d} i\right)^{\frac{1}{1-\eta}} .
$$

Monetary policy We close the model by specifying a simple interest rate rule, pinning down the equilibrium rate of inflation, $\pi_{t} \equiv \log \left(P_{t} / P_{t-1}\right)$. Specifically, we assume that the central bank sets nominal bond prices such that

$$
i_{t}=\phi \pi_{t}
$$

where $\phi>1$ and $i_{t}=-\log \left(Q_{t}\right){ }^{4}$

\footnotetext{
${ }^{4}$ The rule also contains a constant intercept ensuring consistency with the natural rate at the zero-inflation steady state. The term is omitted since it drops out after we log-linearize the model below.
} 


\subsection{Information Structure}

At the core of this paper is an information-robust characterization of equilibria. Throughout, we maintain the assumption of rational expectations, so that conditional on an information set, all expectations are formed using Bayes law. The main novelty is that we do not take a parametric stand on information. Instead we allow for all information structures subject to the following three restrictions.

Assumption 1 (Information bounds). $\Theta_{i, t} \subseteq \mathcal{I}_{i, t} \subseteq \mathcal{I}_{t}^{*}$.

Assumption 1 defines a lower and an upper bound on information available in island $i$ at date $t$. The upper bound, $\mathcal{I}_{t}^{*}$, defines the history of all variables that are realized at date $t$, so that agents cannot learn more than what is potentially knowable under full information. ${ }^{5}$ The lower bound, $\Theta_{i, t}$, includes at least the actions of the agents living in island $i$. Apart from this basic requirement of rationality, $\Theta_{i, t}$ can be specified arbitrarily, allowing the researcher to explore a variety of informational assumptions. As a baseline, we adopt the following specification for $\Theta_{i, t}$ :

$$
\Theta_{i, t}^{\text {baseline }}=\left\{A_{i, t}, C_{i, t}, N_{i, t}, Y_{i, t}, W_{i, t}, \mathcal{I}_{t-\bar{h}-1}^{*}\right\} \cup \Theta_{i, t-1}^{\text {baseline }}
$$

Here we assume that (i) households and firms observe local productivities (and hence outputs) in addition to their own actions and wages, and that (ii) all agents eventually learn the truth at some horizon $\bar{h}+1 \geq 0 .^{6}$

We note that the minimal informational need not necessarily include the full set of publicly available information at time $t$, because agents may not be aware of or use such information in making their decisions, as is generically the case in the literature on rational inattention (e.g., Sims, 2003; Woodford, 2003). In Sections 4.2 and 5, we contrast the baseline with alternative specifications for $\Theta_{i, t}$.

Next, we make the usual assumption that all agents perfectly recall past information.

Assumption 2 (Recursiveness). $\mathcal{I}_{i, t-1} \subseteq \mathcal{I}_{i, t}$.

Finally, we impose ex-ante symmetry across islands and time to streamline the exposition. This does not restrict behavior of the aggregate economy.

\footnotetext{
${ }^{5}$ Notice that which variables are realized at date $t$ is to some extent definitional. In particular, $\mathcal{I}_{t}^{*}$ could contain future innovations if they are realized at date $t$ as in the news literature. In our application, we abstract from this form of news, assuming all innovations to $\left(A_{i, t}, Z_{i, t}\right)$ realize at date $t$.

${ }^{6}$ For now, we do not impose that firms perfectly observe the inverse demand $P_{i, t}$ at the time of making their production choice $Y_{i, t}$. As discussed below, this does not interfere with market clearing. The case where $P_{i, t} \in \Theta_{i, t}$ is discussed in Section 4.2.2.
} 
Assumption 3 (Ex-ante symmetry). The unconditional distribution over $\left(\mathcal{I}_{i, t}, A_{i, t}, Z_{i, t}\right)$ is identical across all $i$ and $t$.

Comment on prices and market clearing It is worth noting that our minimal assumptions do not impose that agents perfectly observe prices, which would be unnecessary restrictive. $^{7}$ This does not mean, however, that agents are completely ignorant of prices, which would prevent prices from clearing markets. To the contrary, imposing market clearing in the parallel primal economy, which we describe in the next section, ensures that agents' equilibrium supply and demand decisions are as responsive to price changes as market clearing requires. As a result, our approach allows us to avoid specifying the details of the signals which agents use to learn about prices without compromising market clearing (see Appendix E for details).

\subsection{Equilibrium Conditions}

We work with a log-linear approximation to the model around the balanced growth path of the economy with no heterogeneity and full information. Lower-case letters denote logdeviations of a variable from the stochastic steady state where $y_{i, t}=a_{t}$ for all $i$ and $\pi_{t}=0$.

The households' Euler equation is given by

$$
c_{i, t}=\mathbb{E}\left[c_{i, t+1}-\phi \pi_{t}+\pi_{t+1} \mid \mathcal{I}_{i, t}\right] .
$$

Combining firms' demand for labor with households' supply, local labor market clearing requires

$$
y_{i, t}=\xi \mathbb{E}\left[x_{i, t} \mid \mathcal{I}_{i, t}\right]+a_{i, t},
$$

where $x_{i, t} \equiv y_{i, t}-c_{i, t}+p_{i, t}-p_{t}$ is the nominal trade-balance on island $i$, and $\xi \equiv 1 /(\zeta+1)$. The linearized price index $p_{t}$ is given by $p_{t}=\int_{0}^{1} p_{i, t} \mathrm{~d} i$. The linearized demand relation and budget constraint take the form

$$
p_{i, t}=\eta^{-1}\left(y_{t}-y_{i, t}\right)+z_{i, t}+p_{t}
$$

\footnotetext{
${ }^{7}$ Limiting the ability of agents to learn from prices most importantly limits their ability to learn about the aggregate state. Lorenzoni (2009) argues that, in practice, learning about aggregates is likely to be impaired by a large number of shocks, model misspecification, and the presence of structural breaks. One specific approach to capture these effects within a simple model like ours would be to decentralize markets so that local prices no longer reflect aggregate states (e.g., Lorenzoni, 2009; Angeletos and La'O, 2013; Chahrour and Ulbricht, 2017). An alternative interpretation is offered by the rational inattention literature (e.g., Maćkowiak and Wiederholt, 2015; Vives and Yang, 2017) where information sets do not reflect all the information that is in principle attainable from prices due to finite processing constraints.
} 
and

$$
\beta b_{i, t}=b_{i, t-1}+x_{i, t}
$$

where $b_{i, t} \equiv B_{i, t} /\left(P_{t} C_{i, t}\right)$ is in levels rather than logs because $B_{i, t}$ can take negative values. Given a process for fundamentals and information $\left\{a_{i, t}, z_{i, t}, \mathcal{I}_{i, t}\right\}$, an equilibrium of the model is a set of processes $\left\{c_{i, t}, y_{i, t}, b_{i, t}, p_{i, t}\right\}$ and $\left\{y_{t}, \pi_{t}\right\}$ that are consistent with (5)-(8), with Bayesian updating, and with market clearing for goods,

$$
y_{t}=\int_{0}^{1} y_{i, t} \mathrm{~d} i=\int_{0}^{1} c_{i, t} \mathrm{~d} i .
$$

(As usual, market clearing for bonds is implied by (8) and (9).)

\section{Information-Robust Characterization}

In this section, we present the methodological innovation in this paper. We begin by defining a fictitious full-information version of our model in which all expectation errors are treated as exogenous wedges. This parallel "wedge economy" can be solved using standard fullinformation tools. We then derive necessary and sufficient conditions on these wedges such that they can be supported as expectation errors in an equilibrium of the incomplete information economy. The set of equilibria in the fictitious wedge-economy satisfying these conditions corresponds to the set of all possible equilibria in the incomplete information economy. Our equivalence result thus provides a tractable method of characterizing all incomplete-information equilibria, without making parametric assumptions about the private information structures of agents.

\subsection{Primal Representation}

Let $\mathbb{E}_{t}[\cdot] \equiv \mathbb{E}\left[\cdot \mid \mathcal{I}_{t}^{*}\right]$ denote the full-information expectations operator. To arrive at the "primal" analogue of the economy characterized in Section 2.3, we replace all expectation operators $\mathbb{E}\left[\cdot \mid \mathcal{I}_{i, t}\right]$ with $\mathbb{E}_{t}[\cdot]+\tau_{i, t}$, where $\left\{\tau_{i, t}\right\}$ are treated as exogenous "expectation" wedges. Specifically, in our case, we replace equations (5) and (6) with the corresponding primal 
equations: ${ }^{8}$

$$
\begin{aligned}
c_{i, t} & =\mathbb{E}_{t}\left[\left(c_{i, t+1}-\tau_{i, t+1}^{c}\right)-\phi \pi_{t}+\pi_{t+1}\right]+\tau_{i, t}^{c} \\
y_{i, t} & =\xi\left(x_{i, t}+\tau_{i, t}^{x}\right)+a_{i, t} .
\end{aligned}
$$

Here $\tau_{i, t}=\left(\tau_{i, t}^{c}, \tau_{i, t}^{x}\right)^{\prime}$ is two-dimensional as there are two expectation operators per information set. The wedges $\tau_{i, t}^{c}$ and $\tau_{i, t}^{x}$ have the interpretation of prediction errors, relative to full-information, regarding household $i$ 's consumption target and island $i$ 's terms-of-trade, $p_{i, t}-p_{t}$, respectively. ${ }^{9}$ Note that both wedges are defined relative to the full-information target that obtains taking as given the behavior of the rest of the economy (i.e., given the expectation errors made on other islands).

\subsection{Characterization Theorem}

We now characterize implementability of the expectation wedges. Let $\mathcal{F}$ denote a stochastic process for the fundamentals $\mathcal{F}_{t} \equiv\left\{\mathrm{d} a_{i, t}, z_{i, t}\right\}_{i \in[0,1]}$, where $\mathrm{d} a_{i, t} \equiv a_{i, t}-a_{i, t-1}$; let $\mathcal{T}$ denote a stochastic process for the expectation wedges $\mathcal{T}_{t} \equiv\left\{\tau_{i, t}^{c}, \tau_{i, t}^{x}\right\}_{i \in[0,1]}$; and let $\mathcal{E}(\mathcal{F}, \mathcal{T})$ denote the set of equilibria in the primal economy induced by $(\mathcal{F}, \mathcal{T})$. We assume the equilibria in $\mathcal{E}(\mathcal{F}, \mathcal{T})$ have a stationary Gaussian distribution that is ex-ante symmetric across islands. ${ }^{10}$ The following theorem states the implementation result.

Theorem 1. Fix $\mathcal{F}, \mathcal{T}$ and $\mathcal{E} \in \mathcal{E}(\mathcal{F}, \mathcal{T})$. Then there exists an information structure consistent with Assumptions 1-3 that implements $\mathcal{T}$, and hence $\mathcal{E}$, in the incomplete-information economy if and only if (i) $\mathbb{E}\left[\tau_{i, t}\right]=0$ and (ii)

$$
\mathbb{E}\left[\tau_{i, t} \theta\right]=0 \text { for all } \theta \in \Theta_{i, t}
$$

hold for all $i$ and $t$.

The theorem gives two conditions that are jointly necessary and sufficient for $\mathcal{T}$ to be implemented by some information structure. Condition (i) is simply the rationality require-

\footnotetext{
${ }^{8}$ Here $\tau_{i, t}^{c}$ is specified after rewriting (5) in its non-recursive form. With this normalization, $\tau_{i, t}^{c}$ defines the gap relative to the optimal level of consumption that household $i$ would choose if it had full information at $t$ and all future dates.

${ }^{9}$ Notice that as $y_{i, t}, c_{i, t} \in \Theta_{i, t}$, all uncertainty about $x_{i, t}$ can be attributed to the terms-of-trade, $p_{i, t}-p_{t}$.

${ }^{10}$ These assumptions can be relaxed. First, in many cases, an appropriate transformation can be used to induce stationarity in the primal economy. E.g., in our case, it suffices to define the primal economy in firstdifferences to ensure stationarity of $\mathcal{E}$ as long as $\mathcal{T}$ is stationary. Second, while we assume $\mathcal{E}$ to be Gaussian, the assumption is not needed when one is only interested in implementing the auto-covariance structure of $\mathcal{E}_{t}$. Finally, ex-ante symmetry is imposed purely for notational convenience. See our general formulation in Appendix A for an extension to non-symmetric cases.
} 
ment that an agent's beliefs cannot be perpetually biased. Condition (ii) is an orthogonality requirement between the expectation wedges and the corresponding lower bounds on information $\Theta_{i, t}$. The necessity of this restriction is the familiar result that expectation errors must be orthogonal to all available information. The novel part of our result is the sufficiency of this condition. For any $\mathcal{E} \in \mathcal{E}(\mathcal{F}, \mathcal{T})$ with $\mathbb{E}\left[\mathcal{T}_{t}\right]=0$, we can always construct an information structure that implements the joint process $\mathcal{E}$ as long as it satisfies (12).

The following example illustrates this in a simple case. The general proof is given in Appendix A and applies to arbitrary linear rational expectation models.

Simple example Consider an economy defined by a single equilibrium condition, $y_{t}=$ $\mathbb{E}\left[a_{t} \mid \mathcal{I}_{t}\right]$, where the fundamental $a_{t}$ satisfies $\mathbb{E}\left[a_{t}\right]=0$, and let $\Theta_{t}=\left\{y_{t-s}\right\}_{s \geq 0}$. The primal economy is given by

$$
y_{t}=a_{t}+\tau_{t}
$$

Let $\mathcal{E}_{t}=\left(y_{t}, a_{t}, \tau_{t}\right)$ be a stationary Gaussian process satisfying (13). Theorem 1 states that for a given process $\mathcal{F}_{t}=a_{t}, \mathcal{E}_{t}$ is implementable by some $\left\{\mathcal{I}_{t}\right\}$, satisfying $y_{t} \in \mathcal{I}_{t}$ for all $t$, if and only if (i) $\mathbb{E}\left[\tau_{t}\right]=0$ and (ii) $\mathbb{E}\left[\tau_{t} y_{t-s}\right]=0$ for all $s \geq 0$. The necessity of conditions (i) and (ii) is immediate, since optimal inference requires that expectation errors are orthogonal to variables in the information set and are unpredictable.

To see why the conditions are also sufficient, suppose that $\mathcal{I}_{t}=\left\{\omega_{t-s}\right\}_{s \geq 0}$ where $\omega_{t}=$ $a_{t}+\tau_{t}$. That is, each period, the agent receives a new signal $\omega_{t}$ that has the same joint distribution over $\left(\omega_{t}, \mathcal{E}_{t}\right)$ as the equilibrium "belief" $y_{t}$ that we wish to implement. Projecting $a_{t}$ onto $y^{t} \equiv\left\{y_{t-s}\right\}_{s \geq 0}$, we have

$$
\mathbb{E}\left[a_{t} \mid \mathcal{I}_{t}\right]=\operatorname{Cov}\left(a_{t}, y^{t}\right)\left[\operatorname{Var}\left(y^{t}\right)\right]^{-1} y^{t}
$$

Notice that

$$
\operatorname{Cov}\left(y_{t}, y^{t}\right)=\left[\begin{array}{llll}
1 & 0 & 0 & \cdots
\end{array}\right] \operatorname{Var}\left(y^{t}\right)
$$

Further notice that (13) in combination with condition (ii) gives $\operatorname{Cov}\left(a_{t}, y^{t}\right)=\operatorname{Cov}\left(y_{t}-\right.$ $\left.\tau_{t}, y^{t}\right)=\operatorname{Cov}\left(y_{t}, y^{t}\right)$. We can thus use (15) to substitute out $\operatorname{Cov}\left(a_{t}, y^{t}\right)$ in (14) to get

$$
\mathbb{E}\left[a_{t} \mid \mathcal{I}_{t}\right]=\left[\begin{array}{llll}
1 & 0 & 0 & \cdots
\end{array}\right] \operatorname{Var}\left(y^{t}\right)\left[\operatorname{Var}\left(y^{t}\right)\right]^{-1} y^{t}=y_{t}
$$

We conclude that as long as conditions (i) and (ii) hold, there exists a simple informationstructure $\left\{\mathcal{I}_{t}\right\}$ that implements $\mathcal{T}_{t}$. Intuitively, observing the equilibrium expectation $y_{t}$ is a sufficient statistic for forming $\mathbb{E}\left[a_{t} \mid \mathcal{I}_{t}\right]$, giving us a simple means of implementing $\mathcal{T}_{t}$. 
Generalization to arbitrary linear economies To streamline the exposition, we have presented Theorem 1 in the context of a specific model economy. However, the proof of the theorem is generic and applies to virtually any linear rational expectations model featured in the literature. Appendix A states the theorem for a general class of linear rational expectations models. The generalized model allows for an arbitrary number of equilibrium conditions involving an arbitrary mix of atomistic and aggregate agents with potentially different decisions, shocks, state variables and information sets.

\section{Inference about the Aggregate Economy}

In this section, we explore how the theoretical restrictions of Theorem 1 translate into restrictions on the behavior of the aggregate economy. In a preliminary step, Section 4.1 derives an aggregate representation of the primal economy, which can be fully described in terms of "macro" wedges $\tau_{t}^{c}=\int_{0}^{1} \tau_{i, t}^{c} \mathrm{~d} i$ and $\tau_{t}^{x}=\int_{0}^{1} \tau_{i, t}^{x} \mathrm{~d} i$. Section 4.2 then maps the restrictions on the "micro" wedges in Theorem 1 to restrictions on the macro wedges that derive from their informational foundation.

\subsection{Aggregation and Equilibrium in the Primal Economy}

We begin with an explicit characterization of equilibrium in the aggregate primal economy. Unlike the aggregate incomplete-information economy, which requires keeping track of the cross-sectional distribution of beliefs, the primal economy permits a simple aggregate representation. Integrating over (10) and (11), we have

$$
\begin{aligned}
& \hat{y}_{t}=\mathbb{E}_{t}\left[\hat{y}_{t+1}-\tau_{t+1}^{c}-\phi \pi_{t}+\pi_{t+1}\right]+\tau_{t}^{c} \\
& \hat{y}_{t}=\xi \tau_{t}^{x}
\end{aligned}
$$

where $\hat{y}_{t} \equiv y_{t}-a_{t}$ is the level of output relative to its (full-information) potential.

Equations (16) and (17) define the aggregate dynamics in the primal economy. Common prediction errors in the Euler equation, captured by $\tau_{t}^{c}$, show up as an Euler equation wedge. Similarly, the common prediction errors regarding the terms-of-trade, captured by $\tau_{t}^{x}$, correspond to the usual definition of the labor wedge. Note that $\tau_{t}^{c}$ and $\tau_{t}^{x}$ are the sole drivers of the aggregate output gap and inflation. If all agents had full information $\left(\tau_{t}^{c}=\tau_{t}^{x}=0\right)$, the aggregate economy would be in its stochastic steady state where output reaches its potential in every period $\left(y_{t}=a_{t}\right)$ and inflation is zero.

In general, a solution for endogenous variables as a function of the joint process $\tau_{t} \equiv$ 
$\left(\tau_{t}^{c}, \tau_{t}^{x}\right)^{\prime}$ can be obtained using standard numerical tools. In our case, a closed-form solution is also available. Substituting out $\hat{y}_{t}$ in (16), $\pi_{t}$ is characterized by the prediction formula

$$
\pi_{t}=\phi^{-1} \mathbb{E}_{t}\left[\xi \mathrm{d} \tau_{t+1}^{x}-\mathrm{d} \tau_{t+1}^{c}+\pi_{t+1}\right]
$$

Following Hansen and Sargent $(1980,1981)$, we obtain an explicit solution for inflation, stated in the following.

Lemma 1. Let $\tau_{t}=A(L) u_{t}$, where $A(L)$ is a square-summable lag polynomial in nonnegative powers of $L$ and the innovations $u_{t}$ are orthogonal white noise. Then there exists a unique stationary equilibrium process for $\left(\hat{y}_{t}, \pi_{t}\right)$, given by

$$
\hat{y}_{t}=\left[\begin{array}{ll}
0 & \xi
\end{array}\right] A(L) u_{t}
$$

and

$$
\pi_{t}=\left[\begin{array}{ll}
-1 & \xi
\end{array}\right] \frac{(1-L) A(L)-\left(1-\phi^{-1}\right) A\left(\phi^{-1}\right)}{\phi L-1} u_{t} .
$$

\subsection{Feasible Dynamics of Aggregate Wedges}

To characterize feasible dynamics of the aggregate economy, we now explore how the restrictions in Theorem 1 constrain the fluctuations of the aggregate wedges $\tau_{t}^{x}$ and $\tau_{t}^{c}$. We begin with the case in which $\Theta_{i, t}$ is given by our baseline specification (4). Alternative specifications for $\Theta_{i, t}$ are considered in Sections 4.2.2-4.2.4.

\subsubsection{Baseline $\Theta_{i, t}$}

To begin, observe that the information set in (4) is informationally equivalent to

$$
\mathcal{S}_{i, t}=\left\{\mathrm{d} c_{i, s}, \mathrm{~d} y_{i, s}, \mathrm{~d} a_{i, s}\right\}_{s=t-\bar{h}}^{t} \cup \mathcal{I}_{t-\bar{h}-1}^{*} .
$$

Here we have used that (i) $n_{i, t}$ and $w_{i, t}$ are linear combinations of $\left(c_{i, t}, y_{i, t}, a_{i, t}\right)$ and are therefore informationally redundant; and (ii) that for any finite horizon $\bar{h}$, observing the sequence of differences $\left\{\mathrm{d} c_{i, s}, \mathrm{~d} y_{i, s}, \mathrm{~d} a_{i, s}\right\}_{s=t-\bar{h}}^{t}$ in addition to $\mathcal{I}_{t-\bar{h}}^{*}$ contains the same information as the corresponding sequence of levels.

To proceed, define $\Delta \tau_{i, t} \equiv \tau_{i, t}-\tau_{t}$ as the idiosyncratic portions of the expectations wedges. Similarly, define $\left(\Delta c_{i, t}, \Delta y_{i, t}\right)$ as the idiosyncratic deviations from aggregate output. By construction the "Delta"-component of any variable is orthogonal to any aggregate variable. Hence, for any two variables $x_{i, t}$ and $y_{i, t}$, we have $\operatorname{Cov}\left[x_{i, t}, y_{i, t}\right]=\operatorname{Cov}\left[x_{t}, y_{t}\right]+\operatorname{Cov}\left[\Delta x_{i, t}, \Delta y_{i, t}\right]$. 
The orthogonality conditions between the wedges $\left(\tau_{i, t}^{c}, \tau_{i, t}^{x}\right)$ and minimal information observables $\left(\mathrm{d} c_{i, t}, \mathrm{~d} y_{i, t}, \mathrm{~d} a_{i, t}\right)$ can then be written as:

$$
\begin{aligned}
\operatorname{Cov}\left[\tau_{t},\left(\mathrm{~d} y_{t-s}, \mathrm{~d} y_{t-s}, \epsilon_{t-s}\right)\right]= & \\
& -\operatorname{Cov}\left[\Delta \tau_{i, t},\left(\Delta \mathrm{d} c_{i, t-s}, \Delta \mathrm{d} y_{i, t-s}, \Delta \mathrm{d} a_{i, t-s}\right)\right] \text { for all } s \geq 0 .
\end{aligned}
$$

Condition (21) requires that any aggregate co-movement on the left-hand side is exactly offset by corresponding "Delta" co-movements on the right-hand side. Our results from the previous section imply that $\mathrm{d} y_{t}$ can be written as a function of $\left(\tau_{t}^{x}, \epsilon_{t}\right)$. Accordingly, the left-hand side is a function of aggregate wedges and productivity shocks only.

To make further progress, we now solve for $\Delta \mathrm{d} c_{i, t}$ and $\Delta \mathrm{d} y_{i, t}$ on the right-hand side of (21). Subtracting $y_{t}$ from both sides of (10) and (11), we obtain

$$
\begin{aligned}
\Delta c_{i, t} & =\mathbb{E}_{t}\left[\Delta c_{i, t+1}-\Delta \tau_{i, t+1}^{c}\right]+\Delta \tau_{i, t}^{c} \\
\Delta y_{i, t} & =\xi\left(x_{i, t}+\Delta \tau_{i, t}^{x}\right)+\Delta a_{i, t} .
\end{aligned}
$$

Together with (7) and (8), conditions (22) and (23) define a (fictitious) small open economy, which pins down the behavior of $\Delta \mathrm{d} c_{i, t}$ and $\Delta \mathrm{d} y_{i, t}$ without need to reference aggregate variables in the economy. In Appendix C.2, we solve the "Delta-economy" in closed form, deriving law of motions for $\Delta \mathrm{d} c_{i, t}$ and $\Delta \mathrm{d} y_{i, t}$ as functions of Delta-fundamentals $f_{i, t} \equiv\left(\Delta a_{i, t}, z_{i, t}\right)$ and Delta-wedges $\Delta \tau_{i, t}$. Using this solution to substitute out $\left(\Delta \mathrm{d} c_{i, t}, \Delta \mathrm{d} y_{i, t}\right)$ on the right-hand side of (21), we obtain the following implementation condition for the macro wedges.

Lemma 2. Fix a (zero mean) $M A(\bar{h})$ process $\tau$ for $\left(\tau_{t}^{c}, \tau_{t}^{x}\right)$ and set $\Theta_{i, t}$ as in (4). Then there exists an information structure consistent with Assumptions 1-3 that implements $\tau$ in the incomplete-information economy, if and only if there exists a (zero mean) $M A(\bar{h})$ process $\Delta \tau$ such that

$$
\Gamma_{s}(\tau, \epsilon)=-\Lambda_{s}(\Delta \tau, f) \quad \text { for all } s \geq 0,
$$

where

$$
\begin{aligned}
\Gamma_{s}(\tau, \epsilon) & \equiv \operatorname{Cov}\left[\tau_{t},\left(\mathrm{~d} y_{t-s}, \mathrm{~d} y_{t-s}, \epsilon_{t-s}\right)\right] \\
\Lambda_{s}(\Delta \tau, f) & \equiv \operatorname{Cov}\left[\Delta \tau_{i, t},\left(\Delta \mathrm{d} c_{i, t-s}, \Delta \mathrm{d} y_{i, t-s}, \Delta \mathrm{d} a_{i, t-s}\right)\right] .
\end{aligned}
$$

The mappings $(\tau, \epsilon) \mapsto \Gamma_{s}$ and $(\Delta \tau, f) \mapsto \Lambda_{s}$ are available in closed-form, stated in (51) and (61) in the appendix.

Lemma 2 provides a general tool to verify implementability of any aggregate wedge pro- 
cess $\tau_{t}$ under various assumptions about the fundamentals $\left\{a_{i, t}, z_{i, t}\right\}$. Specifically, $\tau$ is implementable if and only if it has a finite $\operatorname{MA}(\bar{h})$ representation ${ }^{11}$ and the resulting covariance matrix $\Gamma_{s}(\tau, \epsilon)$ is consistent with the Delta-moments $\Lambda_{s}(\Delta \tau, f)$.

Simple example (continued) To develop an intuition how Theorem 1 maps assumptions on fundamentals to restrictions on expectation wedges, consider again the simple example from above. Theorem 1 requires $\operatorname{Cov}\left[\tau_{t}, y_{t-s}\right]=0$ for all $s \geq 0$ or, after substituting for $y_{t}=a_{t}+\tau_{t}$,

$$
\operatorname{Cov}\left[\tau_{t}, \tau_{t-s}+a_{t-s}\right]=0 \quad \text { for all } s \geq 0
$$

Equation (25) is the simple economy's counterpart to (21). Here the right-hand side is zero, due to the absence of cross-island heterogeneity.

Equation (25) makes specific predictions about the covariance structure between $\tau_{t}$ and $a_{t}$. In particular, evaluating (25) at $s=0$ and noting that by the Cauchy-Schwarz inequality $\operatorname{Cov}\left[a_{t}, \tau_{t}\right] \geq-\sqrt{\operatorname{Var}\left[a_{t}\right] \operatorname{Var}\left[\tau_{t}\right]}$, it follows that the volatility of the expectation wedges is bounded by the fundamental volatility:

$$
\operatorname{Var}\left[\tau_{t}\right] \leq \operatorname{Var}\left[a_{t}\right]
$$

Intuitively, the variance of expectation errors is bounded above by the variance of the forecast target $a_{t}$. In the extreme where $a_{t}$ is constant, there is no scope for confusion, so that $\tau_{t}=0$ in all possible equilibria.

Unrestricted micro-shock benchmark The simple example illuminates how the analogue to Lemma 2 entails restrictions on the volatility and covariance of expectation wedges. Compared to the example, a key difference in the model is the presence of cross-island heterogeneity $\left(\operatorname{Var}\left[f_{i, t}\right]>0\right)$, which can be used to support aggregate fluctuations, even if there are no aggregate shocks. This is because the optimism and pessimism of agents regarding local shocks can be correlated across islands even though the underlying fundamentals are purely idiosyncratic (e.g., Angeletos and La'O, 2013; Benhabib, Wang and Wen, 2015).

As a benchmark, we now review what dynamics are possible if $f_{i, t}$ is treated as a degree of freedom. The case where $f_{i, t}$ is instead fixed at a realistic calibration is studied in Section 5 . By construction, the chosen process for $f_{i, t}$ has no direct impact on the aggregate economy and does not affect the aggregate equilibrium under full information.

\footnotetext{
${ }^{11}$ The restriction to finite MA processes arises, because $\mathcal{I}_{t-h-1}^{*} \subseteq \Theta_{i, t}$ under our baseline specification for $\Theta_{i, t}$. Since all innovation to $\tau_{i, t}$ are part of $\Theta_{i, t}$, the orthogonality requirement then trivially implies that $\tau_{i, t}$ has to have a finite MA representation of order $\bar{h}$.
} 
Proposition 1. Fix a (zero mean) $M A(\bar{h})$ process $\tau$ for $\left(\tau_{t}^{c}, \tau_{t}^{x}\right)$ and set $\Theta_{i, t}$ as in (4). Then for any aggregate productivity process, a, there exists an idiosyncratic process, $f$, such that $\tau$ can be implemented in the incomplete information economy.

The proposition implies that expectation wedges, supported by correlated optimism and pessimism (across islands), can in principle generate any joint process in $\left(\hat{y}_{t}, \pi_{t}\right)$. This generalizes Angeletos and La'O (2013) and Benhabib, Wang and Wen (2015), where expectation errors about local shocks can generate arbitrarily large output variance if the local shocks are sufficiently volatile. Proposition 1 shows that, beyond simply unconditional variances, "sentiment" fluctuations can implement arbitrary processes for $\tau_{t}$ and, by implication, arbitrary autocorrelation structures among the aggregate variables, potentially bypassing all crossequation restrictions that emerge under full information. ${ }^{12}$ Intuitively, expectation errors can easily be correlated, both because information can be correlated between households and firms and because expectation errors by households generally affect both their consumption and labor supply.

\subsubsection{No Demand Uncertainty}

Under the baseline specification for $\Theta_{i, t}$, we make no assumptions regarding how firms and households on island $i$ learn about the inverse demand for the local good, captured by $p_{i, t}$. We now consider the case where $p_{i, t}$ is perfectly observed, so that the inhabitants of island $i$ face no uncertainty about the demand for the local export variety $i$ when production plans are made. That is, information is bounded below by

$$
\Theta_{i, t}=\left\{p_{i, t-s}\right\}_{s \geq 0} \cup \Theta_{i, t}^{\text {baseline }} .
$$

Because local outputs are known, the information contained in $\mathrm{d} p_{i, t}$ is equivalent to

$$
\mathrm{d} p_{i, t}+\eta^{-1} \mathrm{~d} y_{i, t}=\pi_{t}+\theta^{-1} \mathrm{~d} y_{t}+z_{i, t} .
$$

Following similar steps as before, we obtain one additional constraint, which in conjunction with Lemma 2 characterizes the implementation frontier when $p_{i, t} \in \Theta_{i, t}$.

\footnotetext{
${ }^{12}$ In two related contributions, Angeletos, Collard and Dellas (2017) and Ilut and Saijo (2018) illustrate how incomplete information may introduce specific comovement patterns in various variables that differ from full information. However, in contrast to the result in Proposition 1, these comovement patterns are restricted by the specifics of the information-structures considered in these papers, translating into non-trivial cross-equation restrictions.
} 
Lemma 3. Fix a (zero mean) $M A(\bar{h})$ process $\tau$ for $\left(\tau_{t}^{c}, \tau_{t}^{x}\right)$ and set $\Theta_{i, t}$ as in (26). Then there exists an information structure consistent with Assumptions 1-3 that implements $\tau$ in the incomplete-information economy, if and only if there exists a (zero mean) $M A(\bar{h})$ process $\Delta \tau$ such that

$$
\operatorname{Cov}\left[\tau_{t}, \pi_{t-s}+\eta^{-1} \mathrm{~d} y_{t-s}\right]=-\operatorname{Cov}\left[\Delta \tau_{i, t}, z_{i, t-s}\right] \quad \text { for all } s \geq 0 .
$$

holds in addition to (24).

Substituting (28) to (24) for $s=0$, we obtain after some algebra the following restriction on $\operatorname{Var}\left[\hat{y}_{t}\right]$ (see Appendix C.9 for a derivation):

$$
\sqrt{\operatorname{Var}\left[\hat{y}_{t}\right]} \leq \xi \frac{\operatorname{Corr}\left[\hat{y}_{t}, \pi_{t}\right]}{1-\operatorname{Corr}\left[\hat{y}_{t}, \hat{y}_{t-1}\right]} \sqrt{\operatorname{Var}\left[\pi_{t}\right]}
$$

Equation (29) restricts the volatility of $\hat{y}_{t}$ relative to $\pi_{t}$ as a function of its autocorrelation and the contemporaneous correlation with $\pi_{t}$. The following proposition is an immediate corollary.

Proposition 2. Set $\Theta_{i, t}$ as in (26). Then a process $\tau$ for $\left(\tau_{t}^{c}, \tau_{t}^{x}\right)$ with $\operatorname{Var}\left[\tau_{t}^{x}\right]>0$ is implementable in the incomplete information economy only if it implies inflation to be procyclical $\left(\operatorname{Corr}\left[\hat{y}_{t}, \pi_{t}\right]>0\right)$.

To see the logic behind this proposition, recall that

$$
\tau_{t}^{x} \equiv \int_{0}^{1}\left(\mathbb{E}\left[p_{i, t}-p_{t} \mid \mathcal{I}_{i, t}\right]-\left[p_{i, t}-p_{t}\right]\right) \mathrm{d} i=\overline{\mathbb{E}}_{t}\left[p_{i, t}-p_{t}\right] .
$$

To generate an output gap, agents have to make correlated errors regarding the terms of trade, either due to correlated optimism/pessimism regarding the (inverse) demand for the local product, $\overline{\mathbb{E}}_{t}\left[p_{i, t}\right] \neq p_{t}$, as in Benhabib, Wang and Wen (2015) or through a nominal misconception, $\overline{\mathbb{E}}_{t}\left[p_{t}\right] \neq p_{t}$, as in, e.g., Lucas $(1972,1973)$. Imposing $p_{i, t} \in \Theta_{i, t}$ rules out the first possibility, yielding

$$
\tau_{t}^{x}=p_{t}-\overline{\mathbb{E}}_{t}\left[p_{t}\right] .
$$

Because $\mathbb{E}\left[p_{t} \mid \mathcal{I}_{i, t}\right]$ cannot consistently over-predict $p_{t}$ under rational expectations, it follows that $\hat{y}_{t}=\xi \tau_{t}^{x}>0$ is more likely whenever prices are growing, implying that inflation must be procyclical. 


\subsubsection{Public Signals}

Another commonly considered case is that agents learn from public signals about aggregate variables. In general, for any common statistic $s_{t}$, Theorem 1 requires $\operatorname{Cov}\left[\tau_{i, t}, s_{t}\right]=0$. As $\operatorname{Cov}\left[\tau_{i, t}, s_{t}\right]=\operatorname{Cov}\left[\tau_{t}, s_{t}\right]$, it immediately follows that for any $s_{t} \in \Theta_{i, t}$, it must hold that $\operatorname{Cov}\left[\tau_{t}, s_{t}\right]=0$. Augmented by the additional orthogonality requirement, the characterizations in Lemmas 2 and 3 remain fully valid. The following lemma formalizes the result for the baseline case; analog results hold for the alternatives considered above and in Appendix B.

Lemma 4. Fix a (zero mean) $M A(\bar{h})$ process $\tau$ for $\left(\tau_{t}^{c}, \tau_{t}^{x}\right)$ and set

$$
\Theta_{i, t}=\left\{s_{t-s}\right\}_{s \geq 0} \cup \Theta_{i, t}^{\text {baseline }} .
$$

Then there exists an information structure consistent with Assumptions 1-3 that implements $\tau$ in the incomplete-information economy, if and only if

$$
\operatorname{Cov}\left[\tau_{t}, s_{t-s}\right]=0 \quad \text { for all } s \geq 0
$$

and there exists a (zero mean) $M A(\bar{h})$ process $\Delta \tau$ such that (24) holds.

For instance, let $s_{t}=a_{t}$. Then the lemma implies that the aggregate response to productivity shocks must be exactly that of the full information economy. Apart from this restriction, any aggregate wedge process consistent with Lemma 2 continues to be implementable with $a_{t}$ known.

For another illustration, consider the particularly stark case where $s_{t}=\left(u_{t-h}, \epsilon_{t-h}\right)$ with $u_{t}$ being the innovations to $\tau_{t}$ as defined in Lemma 1 . Here the aggregate state is perfectly revealed to agents at some lag $h \leq \bar{h}$. In this case, Lemma 4 requires $\operatorname{Cov}\left[\hat{y}_{t}, \hat{y}_{t-s}\right]=$ $\operatorname{Cov}\left[\hat{y}_{t}, \pi_{t-s}\right]=\operatorname{Cov}\left[\hat{y}_{t}, a_{t-s}\right]=0$ for all $s \geq h$, restricting the autocorrelation of expectationdriven fluctuations within an horizon of $h$ periods. The result generalizes a similar result in Acharya, Benhabib and Huo (2017), which bounds the persistence of a class of sentiment shocks. ${ }^{13}$

\footnotetext{
${ }^{13}$ While the strong conclusions of Lemma 4 may suggest a limited scope of expectation-driven fluctuations in macro variables, such conclusion would likely be premature as information that is in principle publicly available may not be used much by private agents for the purpose of information extraction (e.g, Sims, 2003; Maćkowiak and Wiederholt, 2009). As argued by Woodford (2003), when looking at the information that is actually used by agents to form their beliefs, agents may never share common knowledge - even about signals that have been in the public domain indefinitely.
} 


\subsubsection{Heterogeneous Information Between Households and Firms}

To streamline the exposition, we have so far imposed common knowledge among households and firms within each island. While this assumption is common in the literature, it is arguably unrealistic. In our quantitative exploration, we explore the differential role that household and firm-side errors play for supporting aggregate fluctuations, letting households and firms base their decisions on distinct information sets, $\mathcal{I}_{i, t}^{h}$ and $\mathcal{I}_{i, t}^{f}$, bounded below by

$$
\begin{aligned}
& \Theta_{i, t}^{h}=\left\{C_{i, t}, N_{i, t}, W_{i, t}, \mathcal{I}_{t-\bar{h}}^{*}\right\} \cup \Theta_{i, t}^{h} \\
& \Theta_{i, t}^{f}=\left\{A_{i, t}, N_{i, t}, Y_{i, t}, W_{i, t}, \mathcal{I}_{t-\bar{h}}^{*}\right\} \cup \Theta_{i, t}^{f} .
\end{aligned}
$$

As the theoretical characterization is completely parallel to the one above, we defer the details to Appendix B. Specifically, Lemmas 5 and 6 in the appendix provide characterizations in analog to Lemmas 2 and 3, and Propositions 3 and 4 generalize the results in Propositions 1 and 2 .

\section{Quantitative Analysis}

We now study the quantitative potential for confidence-driven business cycles under an empirically plausible calibration of the fundamental micro-shocks. We conduct two main exercises. First, we compute global upper bounds on confidence-induced output fluctuations, their persistence, and the contemporaneous correlation with inflation. Second, we look at a specific process for the macro wedges, estimated to fit U.S. business cycle data, and ask to what degree those fluctuations are consistent with a theory of incomplete information.

\subsection{Parametrization}

We interpret one period as a quarter. The discount factor $\beta$ is set to 0.99 . The inverse Frisch elasticity $\zeta$ is set to 0.5 , the elasticity of substitution between input varieties $\eta$ is set to 7.5. The monetary policy parameter $\phi$ is set to 1.5 . These values are within the range typically used by the literature.

Next, we set the incomplete information horizon to $\bar{h}=14$ quarters. While we do not have strong priors regarding $\bar{h}$, our choice is consistent with the horizon at which Coibion and Gorodnichenko (2014) find a significant response in professional forecasters' expectation errors to various fundamental and nonfundamental shocks. Later, we also explore the sensitivity of our results to $\bar{h}$, and demonstrate that once the horizon $\bar{h}$ exceeds five periods its 
impact on our results is modest.

It remains to choose processes for the island-specific productivities and demand. We separate local productivities into a permanent component, $\mu_{i, t}$, and a temporary component, $\nu_{i, t}$,

$$
\Delta a_{i, t}=\mu_{i, t}+\nu_{i, t}
$$

where $\nu_{i, t}$ is i.i.d. with zero mean and variance $\sigma_{\nu}^{2}$. The separation ensures that agents can be potentially confused about the precise state of $\Delta a_{i, t}$ (and hence $\mathbb{E}_{t}\left[c_{i, t+1}\right]$ ), even if there are no aggregate productivity shocks. The persistent components $\left\{\mu_{i, t}\right\}$ as well as the local demand shocks $\left\{z_{i, t}\right\}$ follow independent $\operatorname{AR}(1)$ processes with auto-correlations $\left(\rho_{a}, \rho_{z}\right)$ and one-step-ahead variances $\left(\sigma_{a}^{2}, \sigma_{z}^{2}\right)$. The variance and persistence parameters are set based on Foster, Haltiwanger and Syverson (2008), who use plants' price data to disentangle demand from physical productivity shocks at the plant-level. Specifically, we set $\rho_{a}=\rho_{z}=.976, \sigma_{a}=.0552, \sigma_{\nu}=.0478$, and $\sigma_{z}=.2504$, which imply within-product dispersions and quarterly autocorrelations of $z_{i, t}$ and $\Delta a_{i, t}$ that match the corresponding statistics in Foster, Haltiwanger and Syverson (2008). ${ }^{14}$

\subsection{Feasibility frontier}

Our first exercise explores the maximal output volatility — as a function of its persistence and the cyclicality of inflation - that our model can generate via incomplete information about $\left\{\Delta a_{i, t}, z_{i, t}\right\}$. To do so, we focus on the case where there are no aggregate productivity shocks $\left(\operatorname{Var}\left[\epsilon_{t}\right]=0\right)$, ruling out any fundamental source of aggregate fluctuations.

Volatility frontier (definition) Define $\sigma_{\hat{y}}(\tau) \equiv \sqrt{\operatorname{Var}\left[\hat{y}_{t} \mid \mathcal{I}_{t-1}^{*}\right]}$ as the one-step-ahead volatility of output induced by $\tau$, as implied by Lemma 1 . Similarly, define $\rho_{\hat{y}}(\tau) \equiv$ $\operatorname{Corr}\left[\hat{y}_{t}, \hat{y}_{t-1}\right]$ as the first-order autocorrelation of $\hat{y}_{t}$, and define $\gamma_{\hat{y} \pi}(\tau) \equiv \operatorname{Corr}\left[\hat{y}_{t}, \pi_{t}\right]$ as the contemporaneous correlation with inflation. We use Lemma 2 to numerically trace out the volatility frontier for output as a function of its autocorrelation $\rho_{\hat{y}}$ and its contemporaneous correlation with inflation $\gamma_{\hat{y} \pi}$ :

$$
\sigma_{\hat{y}}^{\max }\left(\bar{\rho}_{\hat{y}}, \bar{\gamma}_{\hat{y} \pi}\right) \equiv \max _{\tau, \Delta \tau}\left\{\sigma_{\hat{y}}(\tau)\right\}
$$

\footnotetext{
${ }^{14}$ The underlying calibration targets are .976 and .943 for the quarterly persistence rates of $z_{i, t}$ and $\Delta a_{i, t}$, respectively, and 1.16 and .26 for the (unconditional) within-product dispersions.
} 


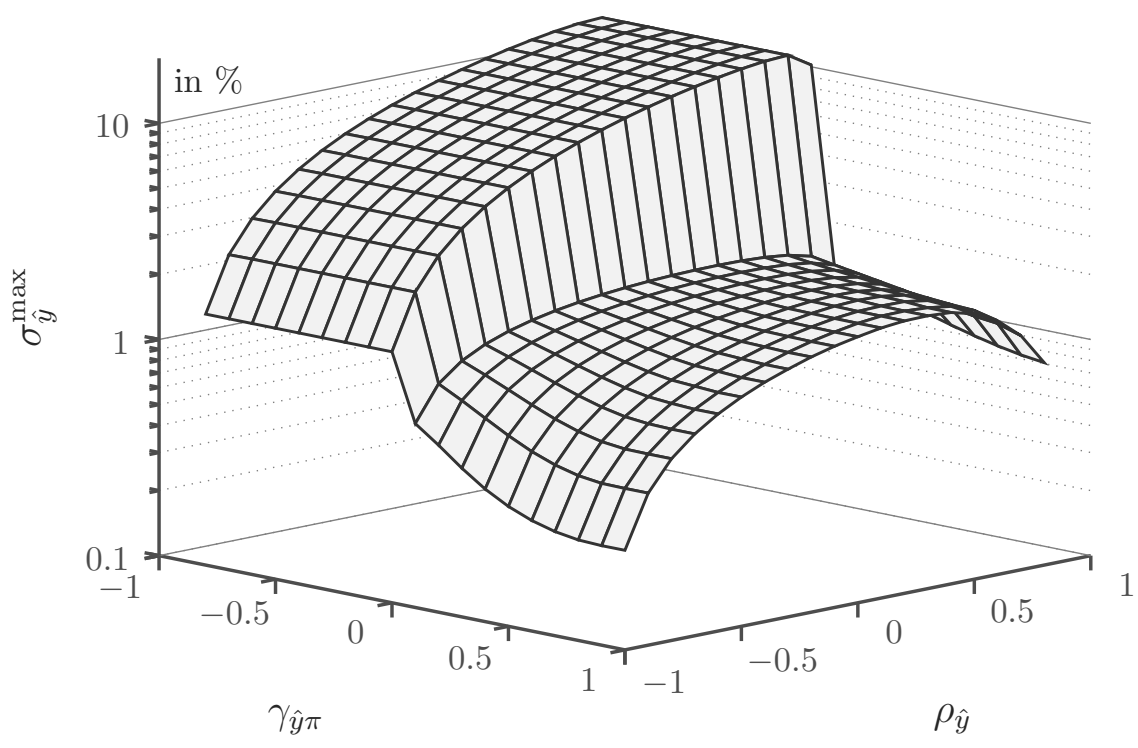

Figure 1: Feasibility frontier. The graph shows the maximal output volatility (denominated in percentage deviations from the balanced growth path) that can be generated by incomplete information as a function of aggregate persistence $\rho_{\hat{y}}$ and the contemporaneous correlation with inflation $\gamma_{\hat{y} \pi}$.

subject to

$$
\begin{aligned}
\Gamma_{s}(\tau, 0) & =-\Lambda_{s}(\Delta \tau, f) \quad \text { for all } s \geq 0 \\
\rho_{\hat{y}}(\tau) & =\bar{\rho}_{\hat{y}} \\
\gamma_{\hat{y} \pi}(\tau) & =\bar{\gamma}_{\hat{y} \pi}
\end{aligned}
$$

where $\Gamma_{s}$ and $\Lambda_{s}$ are defined in Lemma 2, $f=(\Delta a, z)$ is the calibrated process for the idiosyncratic fundamentals, and $\tau$ and $\Delta \tau$ are independent (zero-mean) $\mathrm{MA}(\bar{h})$ processes. ${ }^{15}$

Results for baseline case Figure 1 presents the volatility frontier for $\Theta_{i, t}^{\text {baseline }}$. Here $\sigma_{\hat{y}}^{\max }$ is denominated in percentage deviations from the balanced growth path. The most striking feature is the discrepancy at $\gamma_{\hat{y} \pi}=0$. When inflation is procyclical $\left(\gamma_{\hat{y} \pi}>0\right)$, incomplete information can explain an output volatility up to 1.76 percent. Evaluating $\sigma_{\hat{y}}^{\max }$ at values consistent with U.S. data, $\gamma_{\hat{y} \pi}=.3$ and $\rho_{\hat{y}}=.9$, the maximal volatility amounts to 1.1 percent, which is about $9 / 10$ of the corresponding volatility in the United States. ${ }^{16}$ By contrast, when inflation is countercyclical $\left(\gamma_{\hat{y} \pi}<0\right)$, the maximal volatility is increased by about one order of magnitude.

\footnotetext{
${ }^{15}$ W.l.o.g., the aggregate wedges $\tau$ can be parametrized using two innovations. The island-specific component $\Delta \tau$ loads on up to two innovations in addition to the fundamental shocks that drive $f$.

${ }^{16}$ The comparison is based on the estimation introduced in Section 5.3 and detailed in Appendix D.
} 
The reason for the discrepancy is a fundamental difference in the channels through which the model generates procyclical and countercyclical inflation dynamics. As implied by Proposition 2, countercyclical inflation dynamics are intrinsically tied to expectation errors regarding local demand, which can be quite large for the calibrated process for $z_{i, t}$. By contrast, procyclical inflation dynamics typically require some nominal misconception ${ }^{17}$, which is disciplined by the volatility of aggregate prices that can be generated in the first place.

Micro shocks and macro volatility How do changes in the specification of $\left\{\Delta a_{i, t}\right\}$ and $\left\{z_{i, t}\right\}$ affect the volatility frontier $\sigma_{\hat{y}}^{\max }$ ? To explore the link from micro-shocks to macrovolatility, we conduct comparative statics exercises in $\sigma_{a}, \sigma_{\nu}, \sigma_{z}, \rho_{a}$ and $\rho_{z}$. Here we focus on the empirically plausible case where the macro-correlations $\gamma_{\hat{y} \pi}$ and $\rho_{\hat{y}}$ are fixed at .3 and .9, respectively. The implied volatility frontier, $\sigma_{\hat{y}}^{\max }$, is depicted by the blue dots in Panels $1-5$ of Figure 2. As mentioned above, under the baseline calibration, we have $\sigma_{\hat{y}}^{\max } \approx 1.1$ percent, indicated by the " $\times$ "-marks in the figure.

The sensitivity is strongest in $\sigma_{z}$ and $\rho_{z}$, indicating that correlated expectation errors about the demand shocks $\left\{z_{i, t}\right\}$ are of critical importance for supporting fluctuations in aggregate confidence. In particular, a reduction in $\sigma_{z}$ from its baseline value of 2504 to .01, reduces $\sigma_{\hat{y}}^{\max }$ by a factor of three to .37 percent; an increase in $\sigma_{z}$ to 1.00 , increases $\sigma_{\hat{y}}^{\max }$ to 3.39 percent. Those comparative statics reflect the naturally increasing shape of $\sigma_{\hat{y}}^{\max }$ in any fundamental volatility. Intuitively, the more volatile $z_{i, t}$ (and $a_{i, t}$ ), the larger the potential for agents to make expectation errors, which is a direct consequence of the law of total variance $\left(\operatorname{Var}\left[\mathbb{E}\left\{z_{i, t} \mid \mathcal{I}_{i, t}\right\}\right] \leq \operatorname{Var}\left[z_{i, t}\right]\right)$. In the extreme case where $\sigma_{z} \rightarrow 0$, rationality requires that $\mathbb{E}\left[z_{i, t} \mid \mathcal{I}_{i, t}\right]=0$ for all $t$, even if $\mathcal{I}_{i, t}$ contains no signal about $z_{i, t}$.

Similarly to $\sigma_{z}$, variations in the persistence of $z_{i, t}$ also have a significant impact on $\sigma_{\hat{y}}^{\max }$ : a reduction of $\rho_{z}$ from its baseline value of .976 to .5 , reduces $\sigma_{\hat{y}}^{\max }$ to .35 percent. An increase in the persistence of $z_{i, t}$ to .99 , increases $\sigma_{\hat{y}}^{\max }$ to 3.18. The role of $\rho_{z}$ for supporting expectation errors is two-fold. First, $\operatorname{Var}\left[z_{i, t}\right]$ is increasing in $\rho_{z}$, increasing the potential for expectation errors similar to $\sigma_{z}$. Second, persistence in $z_{i, t}$ (or in $\Delta a_{i, t}$ ), enables optimism and pessimism regarding the wealth of the local household, independently from the direct effects on contemporaneous labor supply and demand. As fluctuations in perceived wealth translate into fluctuations in desired consumption, they can be used to induce pro-cyclical

\footnotetext{
${ }^{17}$ Perceived fluctuations in local demand cannot induce procyclical inflation dynamics because of consumption smoothing. Under standard preferences, consumption typically goes up by less than output in response to a temporary increase in local demand. (This is true as long as $z_{i, t}$ is not too persistent; in our calibration it holds for $\rho_{z} \leq .997$.) The Taylor principle $(\phi>1)$ then implies that booms caused by correlated errors regarding $\left\{z_{i, t}\right\}$ must be accompanied by a drop in inflation so that consumption and output are equilibrated through the induced decline in the real interest rate.
} 

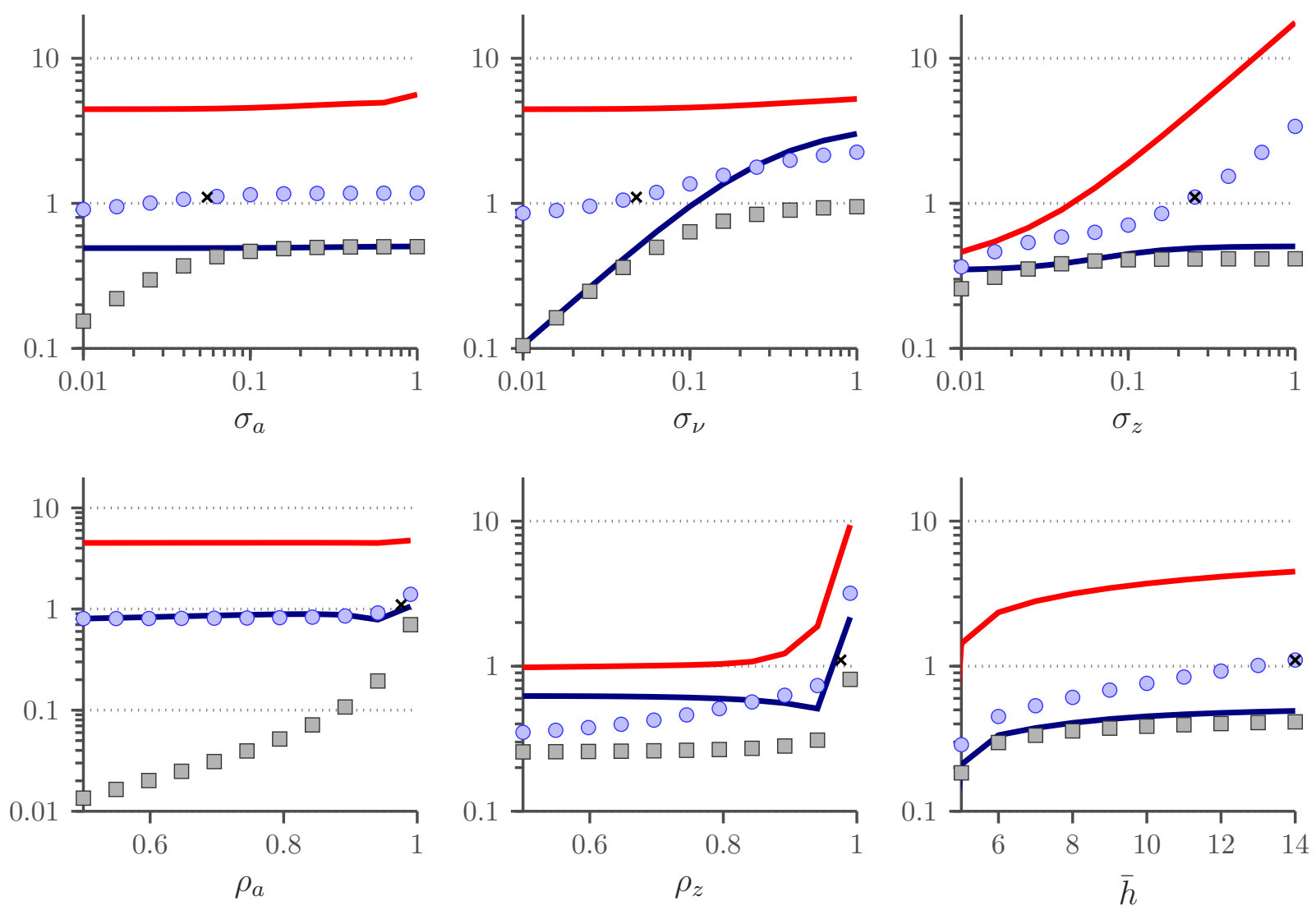

baseline specification for $\Theta_{i, t}$
$\square \quad$ no demand uncertainty
heterogeneous info
heterogeneous info (no demand uncertainty)

Figure 2: Feasibility frontier for alternate specifications of the micro-shocks $\left\{\Delta a_{i, t}, z_{i, t}\right\}$ and for alternate information-bounds $\left\{\Theta_{i, t}\right\}$. The graphs show the maximal output volatility $\sigma_{\hat{y}}^{\max }$ (denominated in percentage deviations from the balanced growth path) that can be generated by incomplete information for the empirically plausible case where $\rho_{\hat{y}}=.9$ and $\gamma_{\hat{y} \pi}=.3$. The " $\times$ "-marks indicate the case where both the micro-shocks and $\Theta_{i, t}$ are fixed at their baseline values shown in Figure 1.

inflation dynamics as in Lorenzoni (2009), which is instrumental for generating the targeted cyclicality of inflation $\left(\gamma_{\hat{y} \pi}=.3\right) .{ }^{18}$

By contrast, variations in the parameters of $\left\{a_{i, t}\right\}$ result in only moderate variations in $\sigma_{\hat{y}}^{\max }$. In particular, reducing $\sigma_{a}$ or $\sigma_{\nu}$ to .01 , implies only marginally smaller values of $\sigma_{\hat{y}}^{\max }$, suggesting that the idiosyncratic productivity shocks $\left\{\Delta a_{i, t}\right\}$ play a somewhat dispensable

\footnotetext{
${ }^{18}$ In order to generate pro-cyclical inflation dynamics through optimism and pessimism about $z_{i, t}$, the information structure must mute the direct substitution effect on labor demand. This can be achieved, for instance, by making agents (sufficiently) informed about $p_{i, t}$ (coupled with some nominal misconception as in Lucas $(1972,1973)$, so that $p_{i, t}$ does not fully reveal $z_{i, t}$ ), which is a sufficient statistic about $\mathbb{E}\left[z_{i, t} \mid \mathcal{I}_{i, t}\right]$ for determining labor demand.
} 
role in our calibration.

Alternative information assumptions We now turn to the sensitivity of the volatility frontier with respect to the lower bounds on information, $\left\{\Theta_{i, t}\right\}$.

As a first alternative, we consider the case without demand uncertainties $\left(p_{i, t} \in \Theta_{i, t}\right)$, as characterized in Lemma 3. Under the baseline parametrization of $\left\{\Delta a_{i, t}, z_{i, t}\right\}$, this reduces $\sigma_{\hat{y}}^{\max }$ to 0.41 , providing a first indication that uncertainty about firms' demand might be relevant to generate sizable confidence-fluctuations. Moreover, compared to $\Theta_{i, t}^{\text {baseline }}$, the sensitivity of $\sigma_{\hat{y}}^{\max }$ in the parameters of $\left\{z_{i, t}\right\}$ is reduced, whereas the sensitivity in the parameters of $\left\{a_{i, t}\right\}$ is heightened (illustrated by the gray squares in Figure 2). This is because $p_{i, t}$ effectively serves as a signal regarding $z_{i, t}$ (see equation (27)), reducing the scope for expectation errors about $z_{i, t}$ and, by implication, increasing the model's reliance on $\Delta a_{i, t}$ for supporting aggregate fluctuations in confidence.

We next relax the assumption that households and firms share the same information set, as characterized in Lemma 5 in the appendix (depicted by the red lines in Figure 2). Relative to $\Theta_{i, t}^{\text {baseline }}$, this increases $\sigma_{\hat{y}}^{\max }$ to 4.49 percent. This reflects the additional flexibility in $\mathcal{I}_{i, t}^{f}$ and $\mathcal{I}_{i, t}^{h}$, due to households not being required to perfectly know the local firm's productivity (i.e., $a_{i, t}, y_{i, t} \notin \Theta_{i, t}^{h}$ ) and firms not being required to perfectly know households' consumption $\left(c_{i, t} \notin \Theta_{i, t}^{f}\right)$. The stark increase in $\sigma_{\hat{y}}^{\max }$ suggests that the common assumption of symmetric information may in fact be quite restrictive.

Finally, we use the heterogeneous information setting to consider the case where only firms have perfect information about $p_{i, t}$, as characterized in Lemma 6 in the appendix (blue lines in Figure 2). Compared to the symmetric-information case without demand uncertainty, $\sigma_{\hat{y}}^{\max }$ is slightly increased to 0.49 . However, the difference between symmetric and heterogeneous information is now much less pronounced, suggesting that imposing informational symmetry is somewhat less restrictive when firms know their demand while making their production choices.

Effects of incomplete-information horizon As a final comparative static, we evaluate the sensitivity of $\sigma_{\hat{y}}^{\max }$ in the incomplete information horizon $\bar{h}$. Because the autocorrelation of any $\mathrm{MA}(\bar{h} \leq 4)$ process is bounded above by less than the targeted autocorrelation $\left(\rho_{\hat{y}}=.9\right)$, we have $\sigma_{\hat{y}}^{\max }=0$ for all $\bar{h} \leq 4$. Conditional on $\bar{h} \geq 5$, the impact of $\bar{h}$ is moderate, especially for the cases without demand uncertainty. Under the baseline specification for $\Theta_{i, t}$, the impact is somewhat more pronounced, reducing $\sigma_{\hat{y}}^{\max }$ to .76 when $\bar{h}$ is reduced to 10 quarters. 


\subsection{Application to U.S. Business Cycles}

We now explore the degree to which U.S. business cycle data is consistent with a theory of incomplete information. To this end, we first estimate an unrestricted wedge process $\hat{\tau}_{t} \equiv\left(\hat{\tau}_{t}^{c}, \hat{\tau}_{t}^{x}\right)$ that in the tradition of Chari, Kehoe and McGrattan (2007) best describes the data. We then partition $\hat{\tau}_{t}$ into an informational component $\tau_{t}^{\text {info }}$ (restricted by our theoretical characterization) and an unrestricted residual component $\tau_{t}^{\text {resid }}$, and maximize the contribution of the informational component $\tau_{t}^{\text {info }}$ under varying assumptions on $\left\{\Delta a_{i, t}, z_{i, t}\right\}$ and $\left\{\Theta_{i, t}\right\}$.

\subsubsection{Methodology}

Here we briefly describe the initial estimation step and then formalize our approach to partitioning the estimated wedge process into an informational and residual component. A detailed description of the preliminary estimation is contained in Appendix D.

Summary of estimation step We use the generalized method of moments (GMM) to estimate the process $\hat{\tau}_{t}$ that best matches the auto-covariance structure of quarterly U.S. data on real per-capita output, inflation, nominal interest rates, and per-capita hours, targeting all auto-covariances between zero and 8 quarters. All moments are computed at business cycle frequencies, applying an high-pass filter with a cutoff of 32 quarters to the model and the data. We model $\hat{\tau}$ as MA(14) processes, which loads on two intrinsic innovations, denoted by $\hat{u}_{t}$, in addition to the productivity shock $\hat{\epsilon}_{t}$.

Despite targeting more data series than there are shocks, the estimated process $\hat{\tau}_{t}$ fits the data quite well: the model replicates the U.S. auto-covariance structure within the confidence bands of the data (see Figure 4 in the appendix). The productivity shock $\hat{\epsilon}_{t}$ explains about 36 percent of the filtered variance in $\hat{y}_{t}$ and about 11 percent to the filtered variance of $y_{t} \cdot{ }^{19}$ The remaining fluctuations are explained by the intrinsic innovations $\hat{u}_{t}$.

Table 1 summarizes key moments of the estimated wedges $\left(\hat{\tau}_{t}^{c}, \hat{\tau}_{t}^{x}\right)$ and the estimated productivity shock $\hat{\epsilon}_{t}$. Most noticeable is the strong positive correlation between the Euler wedge and the labor wedge $\left(\operatorname{Corr}\left[\hat{\tau}_{t}^{c}, \hat{\tau}_{t}^{x}\right]=.99\right)$ and both wedges' negative correlation with productivity growth $\left(\operatorname{Corr}\left[\hat{\tau}_{t}, \hat{\epsilon}_{t}\right]=-.27\right)$.

\footnotetext{
${ }^{19}$ The contribution of $a_{t}$ to $\hat{y}_{t}$ exceeds the one to $y_{t}$, due to a negative correlation between $a_{t}$ and $\hat{y}_{t}$, reflecting a slow adjustment in response to productivity shocks.
} 
Table 1: Summary of estimated U.S. wedges

\begin{tabular}{cccccc}
\hline & & & \multicolumn{3}{c}{ Contemporaneous correlation } \\
\cline { 3 - 6 } & Standard & First-order & with $\hat{\tau}_{t}^{c}$ & with $\hat{\tau}_{t}^{x}$ & with $\hat{\epsilon}_{t}$ \\
\hline deviation & autocorr. & & 1.00 & $\cdot$ \\
\hline$\hat{\tau}_{t}^{c}$ & 0.051 & 0.91 & 0.99 & 1.00 & $\cdot$ \\
$\hat{\tau}_{t}^{x}$ & 0.044 & 0.91 & -0.27 & -0.27 & 1.00 \\
$\hat{\epsilon}_{t}$ & 0.010 & - & & \\
\hline
\end{tabular}

Partitioning of the estimated wedges We partition the estimated wedge process $\hat{\tau}_{t}$ into an informational component $\tau_{t}^{\text {info }}$ and a residual component $\tau_{t}^{\text {resid, }}$

$$
\hat{\tau}_{t}=\tau_{t}^{\text {info }}+\tau_{t}^{\text {resid }}
$$

In parallel to $\hat{\tau}_{t}$, we model both components as statistically independent MA(14) processes,

$$
\begin{aligned}
\tau_{t}^{\text {info }} & =\Phi_{\epsilon}^{\text {info }}(L) \epsilon_{t}^{\text {info }}+\Phi_{u}^{\text {info }}(L) u_{t}^{\text {info }} \\
\tau_{t}^{\text {resid }} & =\Phi_{\epsilon}^{\text {resid }}(L) \epsilon_{t}^{\text {resid }}+\Phi_{u}^{\text {resid }}(L) u_{t}^{\text {resid }}
\end{aligned}
$$

where $\Phi_{\epsilon}^{\text {info }}, \Phi_{u}^{\text {info }}, \Phi_{\epsilon}^{\text {resid }}$ and $\Phi_{u}^{\text {resid }}$ are square-summable lag polynomials in non-negative powers of $L$. The innovations, $\epsilon_{t}^{\text {info }}, \epsilon_{t}^{\text {resid }}, u_{t}^{\text {info }}$ and $u_{t}^{\text {resid }}$, are mutually orthogonal white noise. In particular, $\epsilon_{t}^{\text {info }}$ and $\epsilon_{t}^{\text {resid }}$ are innovations to aggregate productivity, satisfying

$$
\hat{\epsilon}_{t}=\epsilon_{t}^{\mathrm{info}}+\epsilon_{t}^{\mathrm{resid}}
$$

with standard deviations $\sigma_{\epsilon}^{\text {info }}$ and $\sigma_{\epsilon}^{\text {resid }}$. The corresponding lag-polynomial $\Phi_{\epsilon}^{\text {info }}$ captures how incomplete information regarding $a_{t}$ influences the propagation of productivity shocks. ${ }^{20}$ The innovations $u_{t}^{\text {info }}$ and $u_{t}^{\text {resid }}$, each two-dimensional, are intrinsic shocks to $\tau_{t}^{\text {info }}$ and $\tau_{t}^{\text {resid }}$. Accordingly, the lag-polynomial $\Phi_{u}^{\text {info }}$ defines intrinsic fluctuations in $\tau_{t}^{\text {info }}$, driven by expectation errors, whereas $\Phi_{u}^{\text {resid }}$ defines intrinsic fluctuations in the residual wedges $\tau_{t}^{\text {resid }}$.

The defining difference between $\tau_{t}^{\text {info }}$ and $\tau_{t}^{\text {resid }}$ is that we impose the conditions of Theorem 1 on $\tau_{t}^{\text {info }}$, whereas $\tau_{t}^{\text {resid }}$ remains unrestricted. We gauge the potential role of in-

\footnotetext{
${ }^{20}$ Conversely, $\Phi_{\epsilon}^{\text {resid }}$ captures the effects of other potential frictions in propagating productivity shocks. Splitting aggregate productivity into two independent innovations ensures that the volatility generated by incomplete information is independent of the residual wedges $\tau_{t}^{\text {resid }}$. If we instead let $\tau_{t}^{\text {info }}$ and $\tau_{t}^{\text {resid }}$ load jointly on the combined productivity shock $\epsilon_{t}$, we find that one can increase the variance contribution of $u_{t}^{\text {info }}$ almost arbitrarily through incomplete information regarding $a_{t}$ and its propagation through $\tau_{t}^{\text {resid }}$. Below we also consider the case where agents perfectly observe aggregate productivity, in which case both settings give identical results.
} 
complete information for explaining the U.S. business cycle by maximizing the contribution of expectation errors $u_{t}^{\text {info }}$ to the filtered variance of $\hat{y}_{t}$. Let $\hat{y}_{t}^{\mathrm{tfp}} \equiv \mathbb{E}\left[\hat{y}_{t} \mid\left(\epsilon_{t-s}^{\text {info }}, \epsilon_{t-s}^{\text {resid }}\right)_{s \geq 0}\right]$, $\hat{y}_{t}^{\text {info }} \equiv \mathbb{E}\left[\hat{y}_{t} \mid\left(u_{t-s}^{\text {info }}\right)_{s \geq 0}\right]$, and $\hat{y}_{t}^{\text {resid }} \equiv \mathbb{E}\left[\hat{y}_{t} \mid\left(u_{t-s}^{\text {resid }}\right)_{s \geq 0}\right]$ denote the projection of the output gap on aggregate productivity, expectation errors, and residual shocks, respectively. Independence of the innovations implies $\operatorname{Var}\left[\hat{y}_{t}\right]=\operatorname{Var}\left[\hat{y}_{t}^{\mathrm{tfp}}\right]+\operatorname{Var}\left[\hat{y}_{t}^{\text {info }}\right]+\operatorname{Var}\left[\hat{y}_{t}^{\text {resid }}\right]$. Then the maximal contribution of $u_{t}^{\text {info }}$ is given by:

$$
\max _{\tau^{\text {info }, \tau^{\text {resid }}, \sigma_{\epsilon}^{\text {info }}, \sigma_{\epsilon}^{\text {resid }}}}\left\{\operatorname{Var}\left[\hat{y}_{t}^{\text {info }}\right] / \operatorname{Var}\left[\hat{y}_{t}\right]\right\}
$$

subject to two constraints. First, there must exist a (zero-mean) $\mathrm{MA}(\bar{h})$ process for $\left\{\Delta \tau_{i, t}\right\}$ so that the informational component $\tau_{t}^{\text {info }}$ is implementable as characterized in Theorem 1. Second, we require that the auto-covariance structure for $\left(\hat{y}_{t}, \pi_{t}, \epsilon_{t}\right)$ induced by $\left(\tau_{t}^{\text {info }}, \tau_{t}^{\text {resid }}, \epsilon_{t}^{\text {info }}, \epsilon_{t}^{\text {resid }}\right)$ is identical to the one induced by $\left(\hat{\tau}_{t}, \hat{\epsilon}_{t}\right)$. Thus, our partitioned wedges are constrained to produce output, productivity and inflation dynamics that match those observed in the United States.

Observe that $\operatorname{Var}\left[\hat{y}_{t}^{\mathrm{tfp}}\right]$ and $\operatorname{Var}\left[\hat{y}_{t}\right]$ are fully pinned down by the estimated wedge process $\hat{\tau}_{t}$. Hence, instead of maximizing the contribution of $u_{t}^{\text {info }}$ to $\operatorname{Var}\left[\hat{y}_{t}\right]$, we can equivalently maximize the contribution of $u_{t}^{\text {info }}$ to the portion of $\hat{y}_{t}$ that is not driven by the productivity shock, $\operatorname{Var}\left[\hat{y}_{t} \mid\left\{a_{t-s}\right\}_{s \geq 0}\right]=\operatorname{Var}\left[\hat{y}_{t}\right]-\operatorname{Var}\left[\hat{y}_{t}^{\mathrm{tp}}\right]$.

\subsubsection{Results}

The results are presented in Figure 3. To assess which conditions are necessary for incomplete information to generate sizable aggregate fluctuations, we consider five specifications for the lower bounds $\left\{\Theta_{i, t}\right\}$, represented by the five lines in the graph. Along the principal axis, we also consider variations in the parametrization of the micro-shocks $\left\{\Delta a_{i, t}, z_{i, t}\right\}$, scaling their standard deviations, $\left(\sigma_{a}, \sigma_{\nu}, \sigma_{z}\right)$, by up to \pm 1 order of magnitude relative to the baseline calibration. $^{21}$ With the exception of the symmetric information benchmark, all specifications allow households and firms to have access to potentially heterogeneous information as in Section 4.2.4.

Benchmarks As benchmark, we first consider the cases characterized in Lemmas 2 and 5 , where few restrictions are imposed on information beyond rational expectations. In both cases, confidence shocks can fully account for all U.S. business cycle fluctuations unexplained

\footnotetext{
${ }^{21}$ The scaling is applied to all three micro-shocks proportionately to their respective baseline values; i.e., the scaled standard deviations are given by $\left(\sigma_{a}, \sigma_{\nu}, \sigma_{z}\right) \times$ scale.
} 


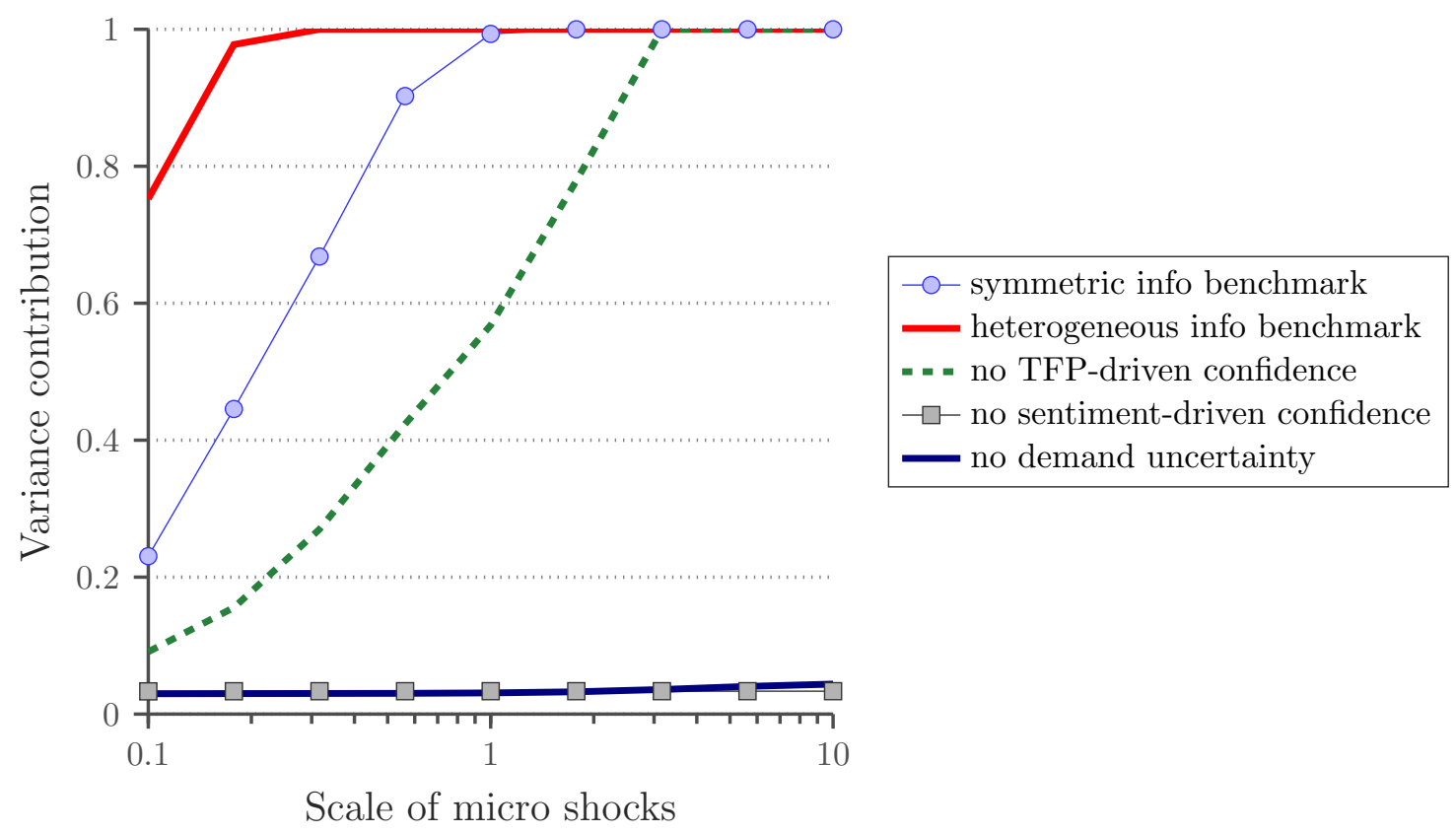

Figure 3: Maximal contribution to U.S. business cycle volatility. The graph shows the maximal variance contribution of $u_{t}^{\text {info }}$ to the portion of the U.S. output gap not driven by productivity, $\operatorname{Var}\left[\hat{y}_{t} \mid\left\{a_{t-s}\right\}_{s \geq 0}\right]$, computed at business cycle frequencies. The lines correspond to different assumptions on the lower bound of information $\left\{\Theta_{i, t}\right\}$. The variation on the principal axis considers alternative values for $\left(\sigma_{a}, \sigma_{\nu}, \sigma_{z}\right)$, which are scaled by up to \pm 1 order of magnitude relative to the baseline calibration (scale $=1$ ).

by the productivity shock $\left(\operatorname{Var}\left[\hat{y}_{t}^{\text {info }}\right] / \operatorname{Var}\left[\hat{y}_{t} \mid\left\{a_{t-s}\right\}_{s \geq 0}\right] \approx 1\right)$, provided that $\left(\sigma_{a}, \sigma_{\nu}, \sigma_{z}\right)$ are at least as volatile as in our baseline calibration (scale $\geq 1$ ). ${ }^{22}$ For the asymmetric information case (red line), the result is also robust to a downward-scaling of the micro-shocks by up to a factor of three. For the symmetric information case (blue dotted line), a reduction in the micro-volatilities by a factor of two (three), reduces the maximal contribution to 90 percent (67 percent).

Sentiments versus noisy learning about aggregate shocks The benchmarks show that, in combination with productivity shocks, rational fluctuations in confidence have the potential to fully account for the U.S. business cycle. We now take a closer look at which type of confidence fluctuations are necessary to achieve this. Specifically, we differentiate between two types of confidence: (i) correlated confidence about idiosyncratic business conditions (aka "sentiment shocks"), and (ii) correlated confidence about aggregate productivity as in Angeletos and La'O (2010) or about future average productivity as in Lorenzoni (2009).

\footnotetext{
${ }^{22}$ Note that this also implies a perfect account of all inflation-dynamics that are unexplained by the productivity shock, since the partitioning of the wedges is constrained to implement the empirical covariance structure for $\left(\hat{y}_{t}, \pi_{t}, \epsilon_{t}\right)$.
} 
First, consider the case of sentiment shocks. We isolate their potential contribution by imposing perfect knowledge about the history of aggregate productivity as in Section 4.2.3, eliminating any scope for TFP-driven fluctuations in confidence. Comparing the resulting contribution (dashed green line) with the benchmark reveals that for small scales of the micro shocks, confidence about aggregate productivity is indeed key for explaining the data. On the other hand, when there is sufficient idiosyncratic volatility (scale $\geq 3$ ), sentiment shocks alone can do as well as the benchmark. For the baseline calibration (scale $=1$ ), sentiment shocks can account for 57 percent of non-productivity fluctuations in U.S. output.

Next, consider the case without sentiment shocks. To eliminate them, we set $\Theta_{i, t}^{f}$ and $\Theta_{i, t}^{h}$ as in (30) and (31), augmented by $\left\{\mu_{i, t-s}, z_{i, t-s}\right\}_{s \geq 0}$. Here we do not include the iidproductivities, $\nu_{i, t}$, in $\Theta_{i, t}^{f}$ or $\Theta_{i, t}^{h}$ as this would allow firms to fully back out $a_{t}$ from observing $a_{i, t}$. However, because $\nu_{i, t}$ is serially uncorrelated and firms know $a_{i, t}$, expectation errors about $\nu_{i, t}$ have no direct effect on their actions, so that all fluctuations in confidence indeed reflect imperfect information about the aggregate productivity state. The quantitative results are shown by the gray squared lines in Figure 3. Under the baseline calibration of the microshocks (scale $=1)^{23}$, TFP-driven fluctuations in confidence can explain at most 3.4 percent of the empirical output volatility, indicating that sentiment-driven fluctuations in confidence are indispensable for explaining the U.S. business cycle with information frictions. This is because aggregate productivity shocks have only a limited importance by themselves, which in turn limits the potential for optimism regarding them to drive the business cycle. ${ }^{24}$

Interestingly, however, the two cases without sentiment- and productivity-driven confidence add up to less than the benchmark, indicating a complementarity between sentiments and confidence about aggregate productivity. Such complementarity may arise, because confidence-fluctuations of one type may serve as noise in endogenous signals regarding the other type of fundamental shock. ${ }^{25}$ Confidence about aggregate productivity shocks may therefore induce additional confidence about local conditions, and visa versa.

No demand uncertainty The final specification considers the case where firms know their demand when making their production choices as characterized in Lemma 6 (solid blue line). In this case, the maximal contribution to the empirical business cycle volatility amounts to 4.1

\footnotetext{
${ }^{23}$ Here we re-calibrate the local productivity shocks to attribute all productivity dispersion to $\nu_{i, t}$. This ensures that the inclusion of $\mu_{i, t}$ in $\Theta_{i, t}^{f}$ and $\Theta_{i, t}^{h}$ does not mechanically reduce the idiosyncratic noise that prevents firms from learning $a_{t}$ from observing $a_{i, t}-\mu_{i, t}=a_{t}+\nu_{i, t}$.

${ }^{24}$ See Chahrour and Jurado (2017) and Angeletos, Collard and Dellas (2018) for independent evidence that productivity shocks play a small role in the business cycle. Indeed, Cochrane (1994) argues that all directly-measurable aggregate shocks play a small role in driving business cycle fluctuations.

${ }^{25}$ See also Chahrour and Gaballo (2016).
} 
Table 2: Implied variance contribution to U.S. output

\begin{tabular}{lccc}
\hline & \multicolumn{3}{c}{ Contribution to } \\
\cline { 2 - 4 } & $\operatorname{Var}\left[y_{t} \mid\left\{a_{t-s}\right\}_{s \geq 0}\right]$ & $\operatorname{Var}\left[y_{t}\right]$ & $\operatorname{Var}\left[\hat{y}_{t}\right]$ \\
\hline Heterogeneous info benchmark & 1.00 & 0.89 & 0.64 \\
Symmetric info benchmark & 0.99 & 0.89 & 0.63 \\
No TFP-driven confidence & 0.57 & 0.51 & 0.36 \\
No sentiment-driven confidence & 0.03 & 0.03 & 0.02 \\
No demand uncertainty & 0.04 & 0.03 & 0.02 \\
\hline
\end{tabular}

Notes. - The table shows the share of output that can be accounted by the intrinsic shocks to the informational component of the estimated wedges, $u_{t}^{\text {info }}$. The contribution of the productivity shock to $\operatorname{Var}\left[y_{t}\right]$ and $\operatorname{Var}\left[\hat{y}_{t}\right]$ is 11 and 36 percent, respectively. All variance contributions are computed at business cycle frequencies for the baseline calibration of $\left\{\Delta a_{i, t}\right\}$ and $\left\{z_{i, t}\right\}$ (i.e., scale $=1$ in Figure 3).

percent, which is almost as low as when fully shutting down all sentiment-fluctuations. The result indicates that demand uncertainties are key for supporting a significant contribution of sentiment-shocks.

Implied variance contribution to U.S. output The results in Figure 3 show the business-cycle contributions to output volatility that is unexplained by productivity, $\operatorname{Var}\left[\tilde{y}_{t} \mid\left\{a_{t-s}\right\}_{s \geq 0}\right]$ (equivalently $\operatorname{Var}\left[y_{t} \mid\left\{a_{t-s}\right\}_{s \geq 0}\right]$ ). Table 2 computes the implied contribution to the overall volatility in $y_{t}$ and $\hat{y}_{t}$. The discrepancy between the three columns reflects the contribution of the productivity shock to $y_{t}$ and $\tilde{y}_{t}$. Looking at the contribution to $y_{t}$, sentiment-driven fluctuations in confidence can account for 51 percent of the empirical volatility. Importantly, however, for a theory of incomplete information to generate significant fluctuations in confidence, firms must face some uncertainty about their idiosyncratic product demands. If this is not the case, then confidence fluctuations can at most explain 3 percent of the empirical volatility in $y_{t}$.

\section{Taking Stock}

We have developed a method to quantify the potential of DSGE models with imperfect information without taking a fully structural stand on the private information of agents. Along the way, we established a conditional equivalence, which holds under the conditions of Theorem 1, between models with dispersed information and a prototype wedge-economy similar to the one in Chari, Kehoe and McGrattan (2007). The informational foundation for these wedges is distinguished from existing theories in its ability to generate arbitrary correlation patterns between these wedges (Proposition 1). Correlated wedges, in turn, are 
critical for the empirical viability of confidence fluctuations because the data imply a strong correlation between the aggregate labor wedge and the Euler wedge. Expectations are a natural candidate for generating the observed correlation, both because information can be correlated between households and firms and because expectation errors by households generally affect both their consumption and labor supply. Our results indicate, however, that two features are crucial to achieve a quantitively important role for such a foundation: (i) micro-shocks must be sufficiently volatile and (ii) idiosyncratic demand must be uncertain at the time of production choices. Our analysis also indicates that observed micro-level volatility is large enough to support substantial aggregate volatility: rational confidence shocks could well explain the data. This suggests to us that future researchers may wish to investigate the empirical evidence regarding (ii), that is, to measure the degree to which firms misperceive their own demand shocks when making their input choices. 


\section{A Representation Theorem}

In this appendix, we present our main representation theorem and show how it applies to the specific model introduced in the main body of the paper.

\section{A.1 General Incomplete Information Model}

Consider a linear economy with $J$ different types of agents. Agents within type $j \in\{0,1, \ldots, J\}$ are indexed by $i \in[0,1]$, but they are ex-ante symmetric: they differ only because of ex-post realizations of shocks. Meanwhile, agents of two different types $j$ and $j^{\prime}$ may be distinguished by differences in their physical environment, differences in their economic objectives, differences in their information, or by their fundamental nature (e.g., households vs. firms). Non-atomistic agents such as a monetary authority can be introduced as part of any type $j$ by including their actions in the aggregate vector, $g_{t}^{a}$ (defined below).

The equilibrium conditions of such a model can be written as

$$
0=\sum_{j=0}^{J} \mathbb{E}\left\{\left[\begin{array}{ll}
\mathbf{A}_{1}^{j} & \mathbf{A}_{2}^{j}
\end{array}\right]\left[\begin{array}{c}
g_{i, t+1} \\
f_{i, t+1}
\end{array}\right]+\left[\begin{array}{ll}
\mathbf{B}_{1}^{j} & \mathbf{B}_{2}^{j}
\end{array}\right]\left[\begin{array}{c}
g_{i, t} \\
f_{i, t}
\end{array}\right] \mid \mathcal{I}_{i, t}^{j}\right\}
$$

with $g_{i, t} \equiv\left[\Delta g_{i, t} ; g_{t}^{a}\right]$ and $f_{i, t} \equiv\left[\Delta f_{i, t} ; f_{t}^{a}\right]$. Here, $\Delta g_{i, t}$ is a $n_{\Delta g^{-}}$dimensional column vector defining purely atomistic variables that satisfy the adding-up constraint $\int_{0}^{1} \Delta g_{i, t} \mathrm{~d} i=0$, whereas $g_{t}^{a}$ is a $n_{g^{a}}$-dimensional column vector defining purely aggregate variables. Similarly, $\Delta f_{i, t}$ and $f_{t}^{a}$ define column vectors of purely atomistic and purely aggregate exogenous Gaussian processes, evolving according to

$$
\begin{aligned}
\Delta f_{i, t} & =f^{i}(L) \epsilon_{i, t} \\
f_{t}^{a} & =f^{a}(L) \epsilon_{t},
\end{aligned}
$$

where again $\int_{0}^{1} \Delta f_{i, t} \mathrm{~d} i=0$. Note that $g_{i, t}$ and $f_{i, t}$ contain variables relevant for any class $j \in J$ (e.g., both household and firm variables), and that $\left(g_{t}^{a}, f_{t}^{a}\right)$ may include but is not restricted to contain the averages corresponding to the variables defined by $\left(\Delta g_{i, t}, \Delta f_{i, t}\right)$. Different types of agents can be subject to different types of shocks, since they can load on different elements in $f_{i, t}$. 


\section{A.2 Primal Representation}

The primal representation of the economy is

$$
0=\left(\sum_{j=0}^{J}\left[\begin{array}{ll}
\mathbf{A}_{1}^{j} & \mathbf{A}_{2}^{j}
\end{array}\right]\right)\left[\begin{array}{l}
\mathbb{E}_{t} g_{i, t+1} \\
\mathbb{E}_{t} f_{i, t+1}
\end{array}\right]+\left(\sum_{j=0}^{J}\left[\begin{array}{ll}
\mathbf{B}_{1}^{j} & \mathbf{B}_{2}^{j}
\end{array}\right]\right)\left[\begin{array}{l}
g_{i, t} \\
f_{i, t}
\end{array}\right]+\sum_{j=1}^{J} \tau_{i, t}^{j}
$$

We denote by $\mathcal{E}(\mathcal{F}, \mathcal{T})$ the set of equilibria of the primal economy with fundamentals $\mathcal{F}_{t} \equiv$ $\left\{\Delta f_{i, t}\right\}_{i \in[0,1]} \cup f_{t}^{a}$ and expectation wedges $\mathcal{T}_{t} \equiv\left\{\tau_{i, t}^{j}\right\}$, which have stationary Gaussian distributions that are ex-ante symmetric across $i{ }^{26}$ The literature offers myriad strategies for solving models of the form in (38). ${ }^{27}$

\section{A.3 Main Theorem}

Before stating the general theorem, we generalize Assumptions 1-3 from the main text to the more general context here.

Assumption 4 (Information bounds). $\left\{\mu_{i, t}^{j}\right\} \subseteq \Theta_{i, t}^{j} \subseteq \mathcal{I}_{i, t}^{j} \subseteq \mathcal{I}_{t}^{*}$ where $\mu_{i, t}^{j}$ defines the "equilibrium expectations" implicit in the primal economy,

$$
\mu_{i, t}^{j} \equiv \mathbb{E}\left[A_{1}^{j} g_{i, t+1}+A_{2}^{j} f_{i, t+1}+B_{1}^{j} g_{i, t}+B_{2}^{j} f_{i, t} \mid \mathcal{I}_{t}^{*}\right]+\tau_{i, t} .
$$

Note that $\mu_{i, t}^{j}$ correspond precisely to agents' equilibrium expectations in the information economy. Accordingly, including $\mu_{i, t}^{j}$ as part of the lower bound $\Theta_{i, t}^{j}$ merely reflects the standard rationality requirement that agents know their own expectations. For instance, the requirement holds in the concrete economy in the main text, since the actions $c_{i, t}, n_{i, t}$ and $y_{i, t}$ encompass the expectations of agents.

Assumption 5 (Recursiveness). $\mathcal{I}_{i, t-1}^{j} \subseteq \mathcal{I}_{i, t}^{j}$ and $\Theta_{i, t}^{j} \subseteq \Theta_{i, t}^{j}$.

The assumption states that all agents perfectly recall past information available to them, which is a standard consistency requirement imposed on dynamic learning models in most economic settings. Our main theorem states conditions so that even with perfect recall, we can construct a consistent information structure to implement $\mathcal{T}_{t}$. The assumption is,

\footnotetext{
${ }^{26}$ Since our assumptions ensure that agents with type- $j$ face ex-ante symmetric environments, any possible asymmetry must pertain to equilibrium outcomes. Such endogenous asymmetries could be accounted by for in our general environment by allowing for equilibrium policies to differ across otherwise identical types $j$.

${ }^{27}$ There is no need for equilibrium in the primal economy to exist or be unique. If $\mathcal{E}(\mathcal{F}, \mathcal{T})$ has multiple elements, our results hold with respect to each of them. Even if $\mathcal{E}(\mathcal{F}, \mathcal{T})$ is a singleton, the incompleteinformation economy may still feature multiplicity as $\mathcal{T}$ itself may be driven by sunspot-realizations as in, e.g., Benhabib, Wang and Wen (2015).
} 
however, not crucial for our results. If one wishes to allow for imperfect recall, an analog version of the proof holds in that case.

Assumption 6 (Ex-ante symmetry). The unconditional distribution over $\left(\mathcal{I}_{i, t}^{j}, f_{i, t}\right)$ is identical across all $i$ and $t$, but not necessarily across $j$.

Compared to the formulation in the main body, the more general formulation of Assumption 6 emphasizes the potential for including ex-ante information differences within the model formulation in (35). In particular, otherwise identical groups of agents who have different information sets can be incorporated by introducing an additional agent type $j^{\prime}$ with an identical economic problem to group $j$, but different information set $\mathcal{I}_{i, t}^{j} \neq \mathcal{I}_{i, t}^{j^{\prime}}$.

Theorem 1 (general version). Fix $\mathcal{F}, \mathcal{T}$ and $\mathcal{E} \in \mathcal{E}(\mathcal{F}, \mathcal{T})$. Then there exists an information structure satisfying Assumptions 4-6 that implements $\mathcal{T}$, and hence $\mathcal{E}$, in the incompleteinformation economy if and only (i) $\mathbb{E}\left[\tau_{i, t}^{j}\right]=0$ and (ii)

$$
\mathbb{E}\left[\tau_{i, t}^{j} \theta\right]=0 \text { for all } \theta \in \Theta_{i, t}^{j} .
$$

hold for all $i, j$, and $t$.

\section{A.4 Proof of Main Theorem}

Consider any expectation wedge $\tau_{i, t}^{j} \in \mathcal{T}_{t}$ from the primal economy and the corresponding lower bound $\Theta_{i, t}^{j}$ on $\mathcal{I}_{i, t}^{j}$ in the incomplete information economy. Define the expectation "targets"

$$
\hat{a}_{i, t}^{j} \equiv A_{1}^{j} g_{i, t+1}+A_{2}^{j} f_{i, t+1}+B_{1}^{j} g_{i, t}+B_{2}^{j} f_{i, t},
$$

which emerge from the equilibrium $\mathcal{E} \in \mathcal{E}(\mathcal{F}, \mathcal{T})$ of the primal economy.

We want to show that conditions (i) and (ii) are jointly necessary and sufficient for the construction of some $\mathcal{I}_{i, t}^{j} \supseteq \Theta_{i, t}^{j}$ such that

$$
\mathbb{E}\left[\hat{a}_{i, t}^{j} \mid \mathcal{I}_{i, t}^{j}\right]=\mathbb{E}\left[\hat{a}_{i, t}^{j} \mid \mathcal{I}_{t}^{*}\right]+\tau_{i, t}^{j} .
$$

When this is true, the actions of agents $(i, j)$ holding information $\mathcal{I}_{i, t}^{j}$ in the information economy will be the same as those of agent $(i, j)$ in the primal economy, sustaining the equilibrium; i.e. a solution to (38) will also be a solution to (35).

To conserve notation, we suppress $(i, j)$ subscripts going forward. 
Necessity. Necessity is immediate, since optimal inference requires that expectation errors are orthogonal to variables in the information set and are unpredictable. To see this, rearrange (40) to get

$$
\tau_{t}=\mathbb{E}\left[\hat{a}_{t} \mid \mathcal{I}_{t}\right]-\mathbb{E}\left[\hat{a}_{t} \mid \mathcal{I}_{t}^{*}\right] .
$$

Computing the unconditional expectation over (41) yields $\mathbb{E}\left[\tau_{t}\right]=0$. Similarly, postmultiplying (41) by $\theta_{t} \in \Theta_{t}$ gives $\mathbb{E}\left[\tau_{t} \theta_{t}\right]=\mathbb{E}\left[\hat{a}_{t} \theta_{t} \mid \mathcal{I}_{t}\right]-\mathbb{E}\left[\hat{a}_{t} \theta_{t} \mid \mathcal{I}_{t}^{*}\right]$ as $\theta_{t} \subseteq \mathcal{I}_{t} \subseteq \mathcal{I}_{t}^{*}$. Again computing the unconditional expectation, we have $\mathbb{E}\left[\tau_{t} \theta_{t}\right]=0$ for all $\theta_{t} \in \Theta_{t}$.

Sufficiency. We demonstrate sufficiency by construction. Let $a_{t} \equiv \mathbb{E}\left[\hat{a}_{t} \mid \mathcal{I}_{t}^{*}\right]$ and consider the information set $\mathcal{I}_{t}=\Theta_{t} \cup\left\{s_{t-\tau}\right\}_{\tau \geq 0}$, where $s_{t} \equiv a_{t}+\tau_{t}=\mu_{t}$ is a signal that replicates the correlation structure of the expectation we wish to implement. Notice that $\mathcal{I}_{t}$ inherits recursiveness from $\Theta_{t}$, ensuring consistency with Assumption 5.

From the law of iterated expectations, we have $\mathbb{E}\left[\hat{a}_{t} \mid s_{t}\right]=\mathbb{E}\left[a_{t} \mid s_{t}\right]$ as $s_{t} \subseteq \mathcal{I}_{t}^{*}$. Projecting $a_{t}$ onto $s_{t}$ we obtain

$$
\begin{aligned}
\mathbb{E}\left[\hat{a}_{t} \mid s_{t}\right] & =\operatorname{Cov}\left[a_{t}, s_{t}\right] \operatorname{Var}\left[s_{t}\right]^{-1} s_{t} \\
& =\operatorname{Cov}\left[s_{t}-\tau_{t}, s_{t}\right] \operatorname{Var}\left[s_{t}\right]^{-1} s_{t} \\
& =\operatorname{Var}\left[s_{t}\right] \operatorname{Var}\left[s_{t}\right]^{-1} s_{t} \\
& =s_{t},
\end{aligned}
$$

where the second line follows from the definition of $s_{t}$ and the third line follows from condition (ii) of the Theorem and the fact that $s_{t}=\mu_{t} \in \Theta_{t}$. Noting that by construction no other $\theta_{t} \in \Theta_{t}$ can improve the forecast about $\hat{a}_{t},{ }^{28}$ we obtain

$$
\mathbb{E}\left[\hat{a}_{t} \mid s_{t}\right]=\mathbb{E}\left[\hat{a}_{t} \mid \mathcal{I}_{t}\right]=\mathbb{E}\left[\hat{a}_{t} \mid \mathcal{I}_{t}^{*}\right]+\tau_{t} .
$$

As the argument above applies to any $\tau_{i, t}^{j} \in \mathcal{T}$, we have constructed exactly the information sets needed to satisfy $(40)$ for all $(i, j, t)$.

\footnotetext{
${ }^{28}$ To see this, note that the forecast error conditional on $s_{t}$ is necessarily uncorrelated with any other $\theta_{t} \in \Theta_{t}: \operatorname{Cov}\left[\hat{a}_{t}-\mathbb{E}\left\{\hat{a}_{t} \mid s_{t}\right\}, \theta_{t}\right]=\operatorname{Cov}\left[\hat{a}_{t}-s_{t}, \theta_{t}\right]=\operatorname{Cov}\left[\hat{a}_{t}-a_{t}-\tau_{t}, \theta\right]=\operatorname{Cov}\left[-\tau_{t}, \theta_{t}\right]=0$. Here the first equality follows from (42); the second one follows per the definition of $\tau_{t}$; the third one follows, because $\hat{a}_{t}-a_{t}$ defines the forecast error under full information $\mathcal{I}_{t}^{*}$, so that any $\theta_{t} \in \Theta_{t} \subset \mathcal{I}_{t}^{*}$ must be orthogonal to it; and the last equality follows from the conditions of the theorem.
} 


\section{A.5 Baseline Economy}

The equilibrium conditions of the model economy can be written in the general form above with $J=\{0,1\}$. Here we use $j=0$ to refer to the full-information set $\left(\mathcal{I}_{i, t}^{0}=\mathcal{I}_{t}^{*}\right)$ and $j=1$ to refer to the information available to households and firms on island $i$. Specifically, let $\Delta g_{i, t}=\left[\Delta c_{i, t}, \Delta y_{i, t}, \Delta p_{i, t}, \Delta b_{i, t-1}\right], g_{t}^{a}=\left[y_{t}, \pi_{t}, p_{t-1}\right]$ and $\Delta f_{i, t}=\left[\Delta z_{i, t}, \Delta a_{i, t}\right]$ and $f_{t}^{a}=\left[a_{t}\right]$. Then the model economy is described by the following system of equations.

For $j=0$ (full information), we have:

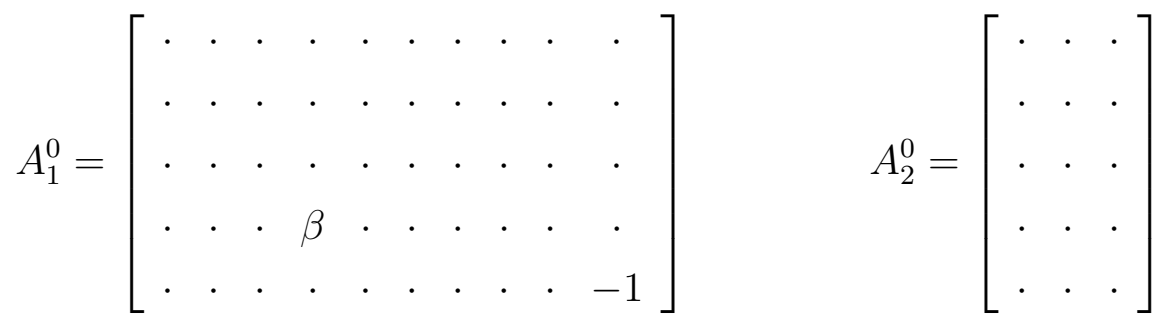

$$
\begin{aligned}
& B_{1}^{0}=\left[\begin{array}{ccccccc}
1 & \cdot & \cdot & \cdot & 1 & \cdot & \cdot \\
\cdot & 1 & . & . & 1 & \cdot & \cdot \\
\cdot & \eta^{-1} & 1 & . & \cdot & \cdot & \cdot \\
1 & -1 & -1 & -1 & \cdot & . & . \\
\cdot & \cdot & . & . & \cdot & 1 & 1
\end{array}\right] \quad B_{2}^{0}=\left[\begin{array}{ccc}
\cdot & \cdot & \cdot \\
\cdot & -1 & -1 \\
-1 & \cdot & \cdot \\
\cdot & \cdot & \cdot \\
\cdot & \cdot & \cdot
\end{array}\right]
\end{aligned}
$$

For the equations describing the expectations of firms and households, we have:

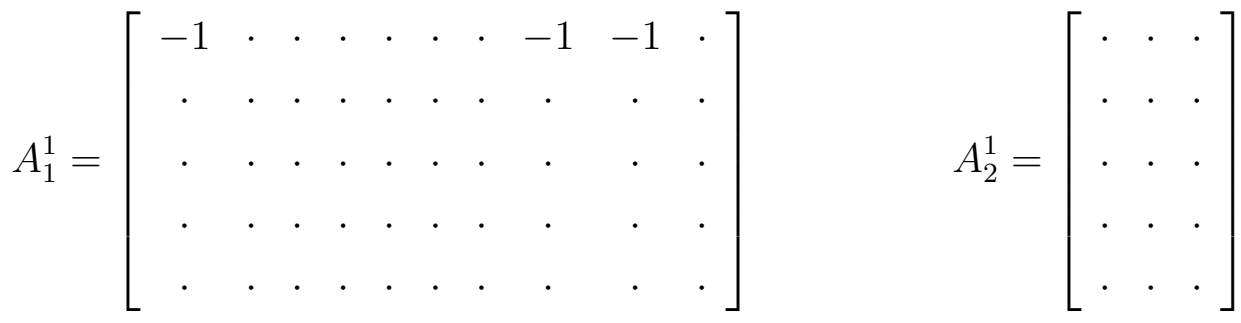

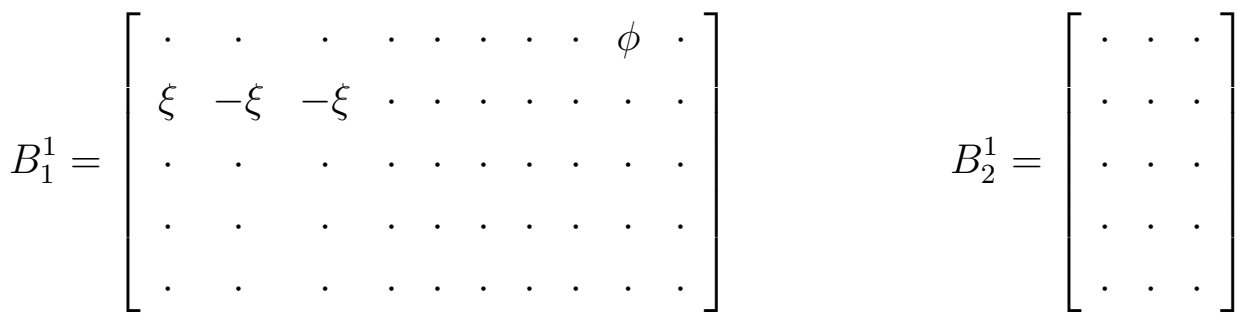

Here, the four equilibrium conditions (5)-(8) are captured by the first four rows in above matrices. The last row defines $\pi_{t}$. Together with the adding-up constraints on $\Delta g_{i, t}$ and $\Delta f_{i, t}$ this completely describes the dynamic system. 


\section{B Heterogeneous Information Between Households and Firms}

This appendix generalizes the theoretical characterization in Section 4.2 for the case where households and firms base their decisions on distinct information sets, $\mathcal{I}_{i, t}^{h}$ and $\mathcal{I}_{i, t}^{f}$.

This distinction between household and firm information sets manifests itself in the labor supply and demand curves, which are given by:

$$
\begin{aligned}
& w_{i, t}=\zeta n_{i, t}+c_{i, t}+\mathbb{E}\left[p_{t} \mid \mathcal{I}_{i, t}^{h}\right] \\
& w_{i, t}=a_{i, t}+\mathbb{E}\left[p_{i, t} \mid \mathcal{I}_{i, t}^{f}\right] .
\end{aligned}
$$

Combing equations, we obtain the same labor market clearing condition as before, stated in (6), but where the "combined" labor wedge is now composed of household and firm errors based on $\mathcal{I}_{i, t}^{h}$ and $\mathcal{I}_{i, t}^{f}$, respectively:

$$
\tau_{i, t}^{x}=\tau_{i, t}^{x, f}-\tau_{i, t}^{x, h}
$$

with

$$
\begin{aligned}
\tau_{i, t}^{x, h} & =\mathbb{E}\left[p_{t} \mid \mathcal{I}_{i, t}^{h}\right]-p_{t} \\
\tau_{i, t}^{x, f} & =\mathbb{E}\left[p_{i, t} \mid \mathcal{I}_{i, t}^{f}\right]-p_{i, t} .
\end{aligned}
$$

Meanwhile, the Euler wedge, $\tau_{i, t}^{c}$, is unambiguously associated with the household's consumption choice and only depends on $\mathcal{I}_{i, t}^{h}$.

As a benchmark, we impose lower bound on $\mathcal{I}_{i, t}^{h}$ and $\mathcal{I}_{i, t}^{f}$ stated in (30) and (31). The corresponding implementation condition for the macro wedges, derived in analog to Section 4.2.1, is given by:

Lemma 5. Fix a (zero mean) $M A(\bar{h})$ process $\tau$ for $\left(\tau_{t}^{c}, \tau_{t}^{x, f}, \tau_{t}^{x, h}\right)$ and set $\Theta_{i, t}^{h}$ and $\Theta_{i, t}^{f}$ as in (30) and (31). Then there exists an information structure consistent with Assumptions 1-3 that implements $\tau$ in the incomplete-information economy, if and only if there exists a (zero mean) $M A(\bar{h})$ process $\Delta \tau$ such that

$$
\begin{aligned}
& \operatorname{Cov}\left[\left(\tau_{t}^{c}, \tau_{t}^{x, h}\right),\left(\mathrm{d} y_{t-s}, \mathrm{~d} \hat{y}_{t-s}, \mathrm{~d} w_{t-s}\right)\right]= \\
& \quad-\operatorname{Cov}\left[\left(\Delta \tau_{i, t}^{c}, \Delta \tau_{i, t}^{x, h}\right),\left(\Delta \mathrm{d} c_{i, t-s}, \Delta \mathrm{d} n_{i, t-s}, \Delta \mathrm{d} w_{i, t-s}\right)\right] \quad \text { for all } s \geq 0
\end{aligned}
$$


and

$$
\begin{aligned}
\operatorname{Cov}\left[\tau_{t}^{x, f},\left(\mathrm{~d} y_{t-s}, \mathrm{~d} \hat{y}_{t-s}, \mathrm{~d} w_{t-s}\right)\right] & = \\
& -\operatorname{Cov}\left[\Delta \tau_{i, t}^{x, f},\left(\Delta \mathrm{d} y_{i, t-s}, \Delta \mathrm{d} n_{i, t-s}, \Delta \mathrm{d} w_{i, t-s}\right)\right] \quad \text { for all } s \geq 0 .
\end{aligned}
$$

The mappings $(\tau, \epsilon) \mapsto(\mathrm{d} y, \mathrm{~d} \hat{y}, \mathrm{~d} w)$ and $(\Delta \tau, f) \mapsto(\Delta \mathrm{d} c, \Delta \mathrm{d} y, \Delta \mathrm{d} n, \Delta \mathrm{d} w)$ are available in closed-form, stated in Appendix C.6.

As (30) and (31) are each a subset of $\Theta_{i, t}^{\text {baseline }}$, the benchmark established in Proposition 1 continues to hold in the current heterogeneous information setting.

Proposition 3. Fix a (zero mean) $M A(\bar{h})$ process $\tau$ for $\left(\tau_{t}^{c}, \tau_{t}^{x}\right)$, and set $\Theta_{i, t}^{h}$ and $\Theta_{i, t}^{f}$ as in (30) and (31). Then for any aggregate productivity process a there exists an idiosyncratic process $f$ such that $\tau$ can be implemented in the incomplete information economy.

Similar to before, we also consider the variation where

$$
\Theta_{i, t}^{f}=\left\{A_{i, t}, P_{i, t}, N_{i, t}, Y_{i, t}, W_{i, t}, \mathcal{I}_{t-\bar{h}}^{*}\right\} \cup \Theta_{i, t}^{f},
$$

so firms face no demand-uncertainty. From (46), it is clear that knowledge of $p_{i, t}$ suffices to fully shut down the firm wedge $\left(\tau_{i, t}^{x, f}=0\right)$. Intuitively, firms only need to know their marginal costs, $w_{i, t}-a_{i, t}$, and their local demand, $p_{i, t}$, to behave as if they have full information (see also Hellwig and Venkateswaran, 2014).

Lemma 6. Fix a (zero mean) $M A(\bar{h})$ process $\tau$ for $\left(\tau_{t}^{c}, \tau_{t}^{x, f}, \tau_{t}^{x, h}\right)$ and set $\Theta_{i, t}^{h}$ and $\Theta_{i, t}^{f}$ as in (30) and (49). Then there exists an information structure consistent with Assumptions 1-3 that implements $\tau$ in the incomplete-information economy, if and only if there exists a (zero mean) $M A(\bar{h})$ process $\Delta \tau$ such that

$$
\tau_{t}^{x, f}=\Delta \tau_{i, t}^{x, f}=0 \quad \text { for all } i, t
$$

holds in addition to (47).

The next proposition generalizes the conclusion in Proposition 2 to the case where only firms learn about $p_{i, t}$.

Proposition 4. Set $\Theta_{i, t}^{h}$ and $\Theta_{i, t}^{f}$ as in (30) and (49). Then an aggregate process $\tau$ for $\left(\tau_{t}^{x}, \tau_{t}^{c}\right)$ with $\operatorname{Var}\left[\tau_{t}^{x}\right]>0$ is implementable in the incomplete information economy only if it implies inflation to be procyclical $\left(\operatorname{Corr}\left[\hat{y}_{t}, \pi_{t}\right]>0\right)$. 
As before, with $p_{i, t}$ known by firms, all output deviations away from potential must be driven by misperception of aggregate nominal conditions, ruling out countercyclical inflation dynamics.

\section{Additional Proofs}

\section{C.1 Proof of Lemma 1}

The characterization for $\hat{y}_{t}$ is immediate. To solve for $\pi_{t}$, let $\pi_{t}=\pi(L) u_{t}$, define

$$
\tilde{A}(L) u_{t} \equiv\left[\begin{array}{ll}
-1 & \xi
\end{array}\right] A(L) u_{t}=\xi \tau_{t}^{x}-\tau_{t}^{c}
$$

and substitute in (18) to obtain

$$
\pi(L) u_{t}=\phi^{-1}\left[\left(L^{-1}-1\right) \tilde{A}(L)+L^{-1} \pi(L)\right]_{+} u_{t}
$$

where $[\cdot]_{+}$sends negative powers of $L$ to zero. Applying the $z$-transform, we obtain the following functional equation

$$
\left(z^{-1}-\phi\right) \pi(z)=\left(1-z^{-1}\right) \tilde{A}(z)+z^{-1} \tilde{A}_{0}+z^{-1} \pi_{0}
$$

Stationarity requires $\pi$ to be analytic on the unit disk (Whiteman, 1983). Evaluating (50) at $z=\phi^{-1} \in(-1,1)$, therefore, pins down

$$
\pi_{0}=\left(1-\phi^{-1}\right) \tilde{A}\left(\phi^{-1}\right)-\tilde{A}_{0}
$$

so that

$$
\pi(z)=\frac{(1-z) \tilde{A}(z)-\left(1-\phi^{-1}\right) \tilde{A}\left(\phi^{-1}\right)}{\phi z-1} .
$$

\section{C.2 Proof of Lemma 2}

From the discussion in the main text, it follows that the following three conditions are jointly necessary and sufficient to invoke Theorem 1:

1. $\mathbb{E}\left[\tau_{t}\right]=\mathbb{E}\left[\Delta \tau_{i, t}\right]=0$,

2. $\tau_{t}$ and $\Delta \tau_{i, t}$ are independent MA processes of at most order $\bar{h}$,

3. condition (24) holds. 
It remains to characterize the mappings $\Gamma_{s}$ and $\Lambda_{s}$.

Characterization of $\Gamma_{s}$ The mapping $\Gamma_{s}$ is immediate from (19),

$$
\Gamma_{s}(\tau, \epsilon)=\xi \operatorname{Cov}\left[\tau_{t}, \mathrm{~d} \tau_{t-s}^{x}\right] \times[1,1,0]+\operatorname{Cov}\left[\tau_{t}, \epsilon_{t-s}\right] \times[1,1,1]
$$

Characterization of $\Lambda_{s}$ We now solve the "Delta-economy" for the endogenous law of motions for $\Delta \mathrm{d} c_{i, t}$ and $\Delta \mathrm{d} y_{i, t}$. The equilibrium of the Delta-economy is defined by (7), (8), (22), (23) together with the definition for $x_{i, t}$ :

$$
\begin{aligned}
\Delta p_{i, t} & =-\eta^{-1} \Delta y_{i, t}+z_{i, t} \\
\beta b_{i, t} & =b_{i, t-1}+x_{i, t} \\
\Delta c_{i, t} & =\mathbb{E}_{t}\left[\Delta c_{i, t+1}-\Delta \tau_{i, t+1}^{c}\right]+\Delta \tau_{i, t}^{c} \\
\Delta y_{i, t} & =\xi\left(x_{i, t}+\Delta \tau_{i, t}^{x}\right)+\Delta a_{i, t} \\
x_{i, t} & =\Delta y_{i, t}-\Delta c_{i, t}+\Delta p_{i, t} .
\end{aligned}
$$

The system can be rewritten as

$$
\begin{aligned}
\mathbb{E}_{t}\left[\mathrm{~d} \Delta y_{i, t+1}\right] & =\delta \mathbb{E}_{t}\left[\xi^{-1} \mathrm{~d} \Delta a_{i, t+1}+\mathrm{d} z_{i, t+1}+\mathrm{d} \Delta \mathrm{d} \tau_{i, t+1}^{x}-\mathrm{d} \Delta \tau_{i, t+1}^{c}\right] \\
\beta b_{i, t} & =b_{i, t-1}+\xi^{-1}\left(\Delta y_{i, t}-\Delta a_{i, t}\right)-\Delta \tau_{i, t}^{x}
\end{aligned}
$$

where $\delta \equiv\left(\eta^{-1}+\xi^{-1}-1\right)^{-1}$, and consumption is determined by

$$
\Delta c_{i, t}=-\delta^{-1} \Delta y_{i, t}+z_{i, t}+\Delta \tau_{i, t}^{x}+\xi^{-1} \Delta a_{i, t}
$$

Fix some process $\left(\Delta \tau_{i, t}^{c}, \Delta \tau_{i, t}^{x}, \Delta a_{i, t}, z_{i, t}\right)^{\prime}=B(L) v_{i, t}$, where $B(L)$ is a square-summable matrix-polynomial in non-negative powers of the lag operator $L$ and the vector $v_{i, t}$ are white noise shocks. Conjecture

$$
\Delta y_{i, t}=\xi(\beta-1) b_{i, t-1}+\Phi(L) v_{i, t}
$$

Substituting (55) in (53), it must be that

$$
\Phi(L) v_{i, t}=\xi \beta \mathrm{d} b_{i, t}+\xi \Delta \tau_{i, t}^{x}+\Delta a_{i, t}
$$

Using (55) to eliminate $\Delta \mathrm{d} y_{i, t+1}$ in (52), we have

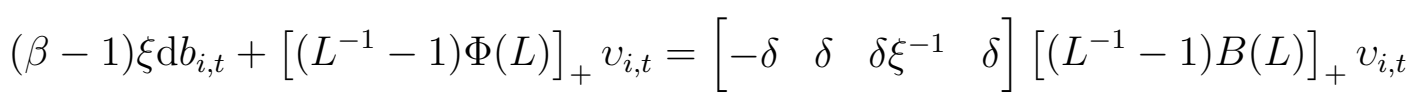


where $[\cdot]_{+}$sends the negative powers of $L$ to zero. Further using (57) to eliminate $\mathrm{d} b_{i, t}$ in (56) and applying the $z$-transform, we obtain the following functional equation

$$
\begin{aligned}
& \left(1-\beta^{-1} z\right) \Phi(z)= \\
& {\left[\begin{array}{llll}
-\delta & \delta & \delta \xi^{-1} & \delta
\end{array}\right]\left[(1-z) B(z)-B_{0}\right]+\Phi_{0}+\left(1-\beta^{-1}\right)\left[\begin{array}{llll}
0 & \xi & 1 & 0
\end{array}\right] B(z) z .}
\end{aligned}
$$

Evaluating (58) at $z=\beta \in(-1,1)$, pins down $\Phi_{0}$ and $\Phi(z)$, from which we obtain the following equilibrium process for $\mathrm{d} \Delta y_{i, t} \equiv \mathrm{d} y(L) v_{i, t}$ and $\mathrm{d} \Delta c_{i, t} \equiv \mathrm{d} c(L) v_{i, t}$ :

$$
\mathrm{d} y(z)=\left[\begin{array}{llll}
-\delta & \delta & \delta \xi^{-1} & \delta
\end{array}\right](1-z) B(z)+\left[\begin{array}{llll}
\delta & \xi-\delta & 1-\delta \xi^{-1} & -\delta
\end{array}\right](1-\beta) B(\beta)
$$

and

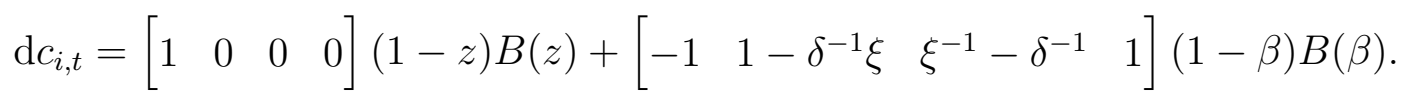

Collecting equations, we obtain

$$
\begin{aligned}
\Lambda_{s}(\Delta \tau, f)=\operatorname{Cov} & {\left[\Delta \tau_{i, t},\left[\begin{array}{cccc}
1 & 0 & 0 & 0 \\
-\delta & \delta & \delta \xi^{-1} & \delta \\
0 & 0 & 1 & 0
\end{array}\right](1-L) B(L) v_{i, t-s}\right] } \\
& +\operatorname{Cov}\left[\Delta \tau_{i, t},\left[\begin{array}{cccc}
-1 & 1-\delta^{-1} \xi & \xi^{-1}-\delta^{-1} & 1 \\
\delta & \xi-\delta & 1-\delta \xi^{-1} & -\delta \\
0 & 0 & 0 & 0
\end{array}\right](1-\beta) B(\beta) v_{i, t-s}\right]
\end{aligned}
$$

for

$$
\Delta \tau_{i, t}=\left[\begin{array}{llll}
1 & 0 & 0 & 0 \\
0 & 1 & 0 & 0
\end{array}\right] B(L) v_{i, t}
$$

\section{C.3 Proof of Proposition 1}

The proposition is proved by construction. In particular, we provide an algorithm that for arbitrary $\left\{\Gamma_{s}\right\}_{s=0}^{\bar{h}}$ constructs processes $\Delta \tau$ and $f$ to satisfy (24).

To begin, substitute (61) to (24), post-multiply both sides by

$$
M \equiv\left[\begin{array}{ccc}
1 & 1 & 0 \\
0 & \delta^{-1} & 0 \\
0 & -\xi^{-1} & 1
\end{array}\right]
$$


and apply the $z$-transform, to obtain the equivalent functional equation

$$
\begin{aligned}
\tilde{\Gamma}(z)=\left[\begin{array}{llll}
1 & 0 & 0 & 0 \\
0 & 1 & 0 & 0
\end{array}\right]\left\{\left[B(z)\left(1-z^{-1}\right) B\left(z^{-1}\right)^{\prime}\right]+\left[\begin{array}{cccc}
1 & 0 & 0 & 0 \\
0 & 1 & 0 & 1 \\
0 & 0 & 1 & 0
\end{array}\right]^{\prime}+\right. \\
+B(z)(1-\beta) B(\beta)^{\prime}\left[\begin{array}{cccc}
-1 & 1-\delta^{-1} \xi & \xi^{-1}-\delta^{-1} & 1 \\
0 & 0 & 0 & 0 \\
0 & 0 & 0 & 0
\end{array}\right]^{\prime}
\end{aligned}
$$

where $\tilde{\Gamma}(z) \equiv \mathcal{Z}\left\{-\Gamma_{s} M\right\}_{s \geq 0}$ is the (one-sided) $z$-transform of $\left\{-\Gamma_{s} M\right\}$, and where $B$ parametrizes the joint process $\left(\Delta \tau_{i, t}, f_{i, t}\right)$ as in the proof of Lemma 2 . In particular, let

$$
B(L)=\left[\begin{array}{l}
B_{\tau}(L) \\
B_{a}(L) \\
B_{z}(L)
\end{array}\right]
$$

where $B_{\tau}(z)$ is a lag-polynomial of size $2 \times n, B_{a}(z)$ and $B_{z}(z)$ are each lag-polynomials of size $1 \times n$, and $n$ is an arbitrary number of innovations. Then (62) can be further rewritten as

$$
\tilde{\Gamma}_{1}(z)+\Omega(z)=\left\{\left(1-z^{-1}\right) B_{\tau}(z) B_{\tau}\left(z^{-1}\right)^{\prime}\right\}_{+}+\Psi(z)+B_{\tau}(z) B_{\tau}(\beta)^{\prime} \Lambda
$$

and

$$
\tilde{\Gamma}_{2}(z)=\left\{\left(1-z^{-1}\right) B_{\tau}(z) B_{a}\left(z^{-1}\right)^{\prime}\right\}_{+},
$$

where $\tilde{\Gamma}_{1}$ and $\tilde{\Gamma}_{2}$ correspond to the first two and third column of $\tilde{\Gamma}$, respectively, and where

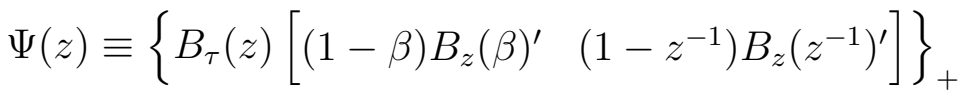

and

$$
\Omega(z) \equiv-(1-\beta)\left(\xi^{-1}-\delta^{-1}\right)\left[B_{\tau}(z) B_{a}(\beta)^{\prime} \quad 0\right]
$$

and

$$
\Lambda \equiv\left[\begin{array}{cc}
-(1-\beta) & 0 \\
(1-\beta)\left(1-\delta^{-1} \xi\right) & 0
\end{array}\right]
$$

Fix $N \leq \bar{h}$ as the largest non-zero power of $z$ in $\tilde{\Gamma}$. Consider the following parametric 
structure for $B_{\tau}, B_{a}$, and $B_{z}$ :

$$
\left[\begin{array}{l}
B_{\tau}(z) \\
B_{a}(z) \\
B_{z}(z)
\end{array}\right]=\left[\begin{array}{cc}
\lambda_{\tau}(z) & I \\
\lambda_{a}(z) & (1-z)^{-1} \lambda_{a, 0} \\
0 & \lambda_{z, 0}+\lambda_{z, 1} z
\end{array}\right]
$$

with

$$
\lambda_{\tau}(z)=\left[\begin{array}{lll}
\lambda_{\tau, 1}+\rho z & \cdots & \lambda_{\tau, N}+\rho^{N} z^{N}
\end{array}\right]
$$

and

$$
\lambda_{a}(z)=\left[\begin{array}{lll}
(1-z)^{-1} \lambda_{a, 1} & \cdots & (1-z)^{-1} \lambda_{a, N}
\end{array}\right],
$$

and where $\left\{\lambda_{a, j}, \lambda_{z, j}\right\}$ are of size $1 \times 2$ and $\left\{\lambda_{\tau, j}\right\}$ are of size $2 \times 2$. Observe that $B_{\tau}$ is at most of order $\bar{h}$ in line with the requirements of Lemma 2.

Condition (64) then simplifies to

$$
\tilde{\Gamma}_{2}(z)=\lambda_{\tau}(z) \lambda_{a}^{\prime}+\lambda_{a, 0}^{\prime}
$$

So for any $\lambda_{\tau}$, it suffices to set

$$
\begin{aligned}
& \lambda_{a, s}=\rho^{-s} \tilde{\Gamma}_{2, s}^{\prime} \quad \forall s \geq 1, \quad \text { and } \\
& \lambda_{a, 0}=\tilde{\Gamma}_{2,0}^{\prime}-\sum_{j=1}^{N} \lambda_{\tau, j}^{\prime} \lambda_{a, j}
\end{aligned}
$$

in order to satisfy orthogonality with respect to $a_{i, t}$.

Regarding condition (63), we have that

$$
\Pi(z) \equiv \tilde{\Gamma}_{1}(z)+\Omega(z)-\Lambda-I=\left\{\left(1-z^{-1}\right) \tau_{\tau}(z) \tau_{\tau}\left(z^{-1}\right)^{\prime}\right\}_{+}+\Psi_{0}+\lambda_{\tau}(z) \lambda_{\tau}(\beta)^{\prime} \Lambda
$$

where

$$
\Omega(z)=-\tilde{\Gamma}_{2}(z)\left[\begin{array}{ll}
\left(\xi^{-1}-\delta^{-1}\right) & 0
\end{array}\right]
$$

and

$$
\Psi_{0} \equiv \Psi(z)=\left[(1-\beta)\left(\lambda_{z, 0}^{\prime}+\beta \lambda_{z, 1}^{\prime}\right) \quad \lambda_{z, 0}^{\prime}\right]
$$

Notice that (i) the left-hand side, $\Pi(z)$, is exogenously determined by the aggregate economy that we are trying to implement, and (ii) we have $\Psi_{0}$ as a degree of freedom to induce an 
arbitrary unconditional covariance on the right-hand side. Writing out the right-hand side in the time-domain, we have

$$
\begin{aligned}
& \Pi_{0}=\Psi_{0}-\rho \lambda_{\tau, 1}^{\prime}+\frac{\rho^{2}}{1-\rho^{2}}+\sum_{j=1}^{N} \lambda_{\tau, j} \lambda_{\tau, j}^{\prime}(I+\Lambda)+\sum_{j=1}^{N} \rho^{j} \beta^{j} \lambda_{\tau, j} \Lambda \\
& \Pi_{s}=\rho^{s} \lambda_{\tau, s}^{\prime}(I+\Lambda)-\rho^{s+1} \lambda_{\tau, s+1}^{\prime}+\rho^{2 s} \beta^{s} \Lambda .
\end{aligned}
$$

Initialized at $\lambda_{N+1}=0,(66)$ can be solved recursively backwards for a sequence $\left\{\lambda_{\tau, s}\right\}$ that ensures orthogonality with respect to $\left(c_{i, t-s}, y_{i, t-s}\right)_{s \geq 1}$. Finally, orthogonality with respect to $\left(c_{i, t}, y_{i, t}\right)$ is achieved by setting $\Psi_{0}$ to satisfy (65), completing the proof.

\section{C.4 Proof of Lemma 3}

The result follows from Theorem 1 and Lemma 2. See the discussion in the main text for details.

\section{C.5 Proof of Proposition 2}

The result is a corollary to Proposition 4, which is derived under a less restricting lower bound on information.

\section{C.6 Proof of Lemma 5}

Condition (47) is immediate from (30). Similarly, condition (48) is immediate after noticing that $a_{i, t}$ is a linear combination of $y_{i, t}$ and $n_{i, t}$ and can, therefore, be omitted from (31). It follows that in analog to Lemma 2, conditions (30) and (31) together with $\mathbb{E}\left[\tau_{t}\right]=\mathbb{E}\left[\Delta \tau_{i, t}\right]=0$, and $\tau_{t}$ and $\Delta \tau_{i, t}$ being independent MA processes of at most order $\bar{h}$ are jointly necessary and sufficient to invoke the conditions of Theorem 1.

It remains to characterize the mappings $(\tau, \epsilon) \mapsto(\mathrm{d} y, \mathrm{~d} \hat{y}, \mathrm{~d} w)$ and $(\Delta \tau, f) \mapsto(\Delta \mathrm{d} c, \Delta \mathrm{d} y$, $\Delta \mathrm{d} n, \Delta \mathrm{d} w)$.

Characterization of aggregate variables The law of motions for $\mathrm{d} y_{t}$ and $\mathrm{d} \hat{y}_{t}$ are immediate from Lemma 1. The law of motion for $\mathrm{d} w_{t}$ follows from (44),

$$
\mathrm{d} w_{t}=\epsilon_{t}+\pi_{t}+\mathrm{d} \tau_{t}^{x, f}
$$

where $\pi_{t}$ is again characterized in Lemma 1. 
Characterization of Delta-variables The law of motions for $\Delta \mathrm{d} y_{i, t}$ and $\Delta \mathrm{d} c_{i, t}$ are already characterized in (59) and (60). Subtracting d $w_{t}$ from (43) yields

$$
\Delta \mathrm{d} w_{i, t}=\zeta \Delta \mathrm{d} n_{i, t}+\Delta \mathrm{d} c_{i, t}+\Delta \mathrm{d} \tau_{i, t}^{h}
$$

Finally, subtracting $y_{t}$ from (1), we get

$$
\Delta \mathrm{d} n_{i, t}=\Delta \mathrm{d} y_{i, t}-\Delta \mathrm{d} a_{i, t}
$$

\section{C.7 Proof of Proposition 3}

The result is a corollary to Proposition 1, which is derived under a more restricting lower bound on information.

\section{C.8 Proof of Lemma 6}

The result follows from Theorem 1 and Lemma 5. See the discussion in the main text for details.

\section{C.9 Proof of Proposition 4}

From Lemma 6, we have that $\tau_{i, t}^{x, f}=0$ implying $\tau_{i, t}^{x}=-\tau_{i, t}^{x, h}$. Substituting in (47), combining with (67)-(69), and evaluating at $s=0$, yields

$$
\begin{aligned}
\operatorname{Cov}\left\{\left[\begin{array}{c}
\tau_{t}^{c} \\
-\tau_{t}^{x}
\end{array}\right],\left[\begin{array}{c}
\xi \mathrm{d} \tau_{t}^{x}+\epsilon_{t} \\
\xi \mathrm{d} \tau_{t}^{x} \\
\pi_{t}+\epsilon_{t}
\end{array}\right]\right\}= \\
\quad-\operatorname{Cov}\left\{\left[\begin{array}{c}
\Delta \tau_{i, t}^{c} \\
-\Delta \tau_{i, t}^{x}
\end{array}\right],\left[\begin{array}{c}
\Delta \mathrm{d} c_{i, t} \\
\left(\xi^{-1}-1\right)\left(\Delta \mathrm{d} y_{i, t}-\Delta \mathrm{d} y_{i, t}-\Delta \mathrm{d} a_{i, t}\right)+\Delta \mathrm{d} c_{i, t}-\Delta \mathrm{d} \tau_{i, t}^{x}
\end{array}\right]\right\} .
\end{aligned}
$$

Pre-multiplying both sides by $[0,1]$ and post-multiplying by $\left[-1,1-\xi^{-1}, 1\right]^{\prime}$, we get

$$
\operatorname{Cov}\left[\tau_{t}^{x}, \mathrm{~d} \tau_{t}^{x}-\pi_{t}\right]=-\operatorname{Cov}\left[\Delta \tau_{i, t}^{x}, \Delta \mathrm{d} \tau_{i, t}^{x}\right]
$$


Observe that for any $\operatorname{Corr}\left[\Delta \tau_{i, t}^{x}, \Delta \tau_{i, t-1}^{x}\right] \in[-1,1]$ the right-hand side is weakly negative. Hence it must hold that

$$
\operatorname{Cov}\left[\tau_{t}^{x}, \mathrm{~d} \tau_{t}^{x}-\pi_{t}\right]=\operatorname{Var}\left[\tau_{t}^{x}\right]-\operatorname{Cov}\left[\tau_{t}^{x}, \tau_{t-1}^{x}\right]-\operatorname{Cov}\left[\tau_{t}^{x}, \pi_{t}\right] \leq 0
$$

Multiplying by $\xi^{2}$ and rearranging yields (29) in the main text. The proposition follows after noting that for any $\operatorname{Var}\left[\tau_{t}^{x}\right]>0$ the left-hand side of $(29)$ is strictly positive, whereas the right-hand side is non-positive when $\operatorname{Corr}\left[\hat{y}_{t}, \pi_{t}\right] \leq 0$.

\section{C.10 Proof of Lemma 4}

The result follows from Theorem 1 and Lemma 2. See the discussion in the main text for details.

\section{Estimation of Unrestricted Wedge Process}

Here we describe the methodology for estimating the unrestricted wedges $\hat{\tau}_{t}$ used in Section 5.3.

\section{D.1 Description of Methodology}

We model the unrestricted wedges as a MA(14) process, which loads on two intrinsic innovations, represented by the $2 \times 1$ vector $u_{t}$, in addition to the productivity shock $\epsilon_{t}$,

$$
\tau_{t}=\Phi_{\epsilon}(L) \epsilon_{t}+\Phi_{u}(L) u_{t}
$$

where $\Phi_{\epsilon}(L)$ and $\Phi_{u}(L)$ are square-summable lag polynomials in non-negative powers of $L$, and $\epsilon_{t}$ and $u_{t}$ are orthogonal white noise. W.l.o.g., we normalize $\operatorname{Var}\left[u_{t}\right]=I_{2}$, leaving us to estimate $\gamma_{\mathrm{ma}} \equiv\left(\Phi_{\epsilon}, \Phi_{u}, \sigma_{\epsilon}\right)$. For this purpose, we use the generalized method of moments (GMM) to minimize the distance between the model's covariance structure and U.S. data on real per-capita output, inflation, nominal interest rates, and per-capita hours. ${ }^{29}$ Let

$$
\tilde{\Omega}_{T}=\operatorname{vech}\left\{\operatorname{Var}\left[\left(\tilde{q}_{t}^{\text {data }}, \ldots, \tilde{q}_{t-k}^{\text {data }}\right)\right]\right\},
$$

\footnotetext{
${ }^{29}$ Data range from 1960Q1 to 2012Q4. Real output is given by nominal output divided by the GDP deflator. Inflation is defined as the log-difference in the GDP deflator. Interest rates are given by the Federal Funds Effective rate. Hours are given by hours worked in the non-farm sector. Variables are put in per-capita terms using the non-institutional population over age 16.
} 
denote the empirical auto-covariance matrix of frequency-filtered quarterly US data for $q \equiv$ $\left(y_{t}, \pi_{t}, i_{t}, n_{t}\right)$. We target auto-covariances between zero and $k=8$ quarters. For the filtering, we use the Baxter and King (1999) approximate high-pass filter with a truncation horizon of 32 quarters, which we denote by $\tilde{q}_{t} \equiv B K_{32}\left(q_{t}\right){ }^{30}$

To conserve on the 91 parameters that characterize $\gamma_{\mathrm{ma}}$, we make two observations, documented in Figure 4 below. First, $\tilde{\Omega}_{T}$ is well-described by a $\operatorname{VAR}(1)$ process for $\tau_{t}$. Second, a $\operatorname{MA}(14)$ truncation of the $\operatorname{VAR}(1)$ process that best replicates $\tilde{\Omega}_{T}$ is almost indistinguishable (in terms of second moments) from the $\operatorname{VAR}(1)$ process itself. Accordingly, we construct $\gamma_{\text {ma }}$ by first estimating $\tau_{t}$ as a $\operatorname{VAR}(1)$ that is driven by $u_{t}$ and $\epsilon_{t}$, and then constructing $\hat{\gamma}_{\text {ma }}$ as the MA(14) truncation of the estimated process.

Let $\gamma_{\text {ar }}$ denote the 10 parameters characterizing the $\operatorname{VAR}(1)$ and $\sigma_{\epsilon}$. Then the estimator is given by

$$
\hat{\gamma}_{\mathrm{ar}}=\underset{\gamma_{\mathrm{ar}}}{\operatorname{argmin}}\left(\tilde{\Omega}_{T}-\tilde{\Omega}\left(\gamma_{\mathrm{ar}}\right)\right)^{\prime} W^{-1}\left(\tilde{\Omega}_{T}-\tilde{\Omega}\left(\gamma_{\mathrm{ar}}\right)\right),
$$

where $\tilde{\Omega}\left(\gamma_{\mathrm{ar}}\right)$ is the model analogue to $\tilde{\Omega}_{T}$ and $W$ is a diagonal matrix with the bootstrapped variances of $\tilde{\Omega}_{T}$ along the main diagonal. To avoid the issues detailed in Gorodnichenko and $\mathrm{Ng}$ (2009), our model analogue $\tilde{\Omega}\left(\gamma_{\mathrm{ar}}\right)$ is computed after applying the same filtering procedure to the model that we have applied to the data.

A final challenge for estimating the model is that filtering the model can be computational expensive. We address this issue by proving the following equivalence results.

Lemma 7. Estimator (70) is equivalent to

$$
\hat{\gamma}_{\mathrm{ar}}=\underset{\gamma_{\mathrm{ar}}}{\operatorname{argmin}}\left(\Omega_{T}-\Omega\left(\gamma_{\mathrm{ar}}\right)\right)^{\prime} \tilde{W}^{-1}\left(\Omega_{T}-\Omega\left(\gamma_{\mathrm{ar}}\right)\right),
$$

where $\Omega \equiv \operatorname{vech}\left\{\operatorname{Var}\left[\left(\mathrm{d} s_{t}, \ldots, \mathrm{d} s_{t-K}\right)\right]\right\}$ and $\tilde{W} \equiv\left(\Xi^{\prime} W^{-1} \Xi\right)^{-1}$ for $K=k+2 \bar{\tau}$. The transformation matrix $\Xi$ is defined in (76).

The lemma establishes an exact equivalence (as opposed to an asymptotic equivalence) between the original GMM estimator (70) and an alternative estimator where the unfiltered model is estimated (in first differences) on unfiltered data and the filtering is achieved by replacing $W$ with $\tilde{W}$. Using (71) in place of (70), estimation becomes straightforward as the mapping from $\gamma_{\text {ar }}$ to $\Omega\left(\gamma_{\text {ar }}\right)$ is available in closed form.

\footnotetext{
${ }^{30}$ The Baxter and King (1999) filter requires specification of a lag-length $\bar{\tau}$ for the approximation. We set $\bar{\tau}$ to their recommended value of 12 .
} 

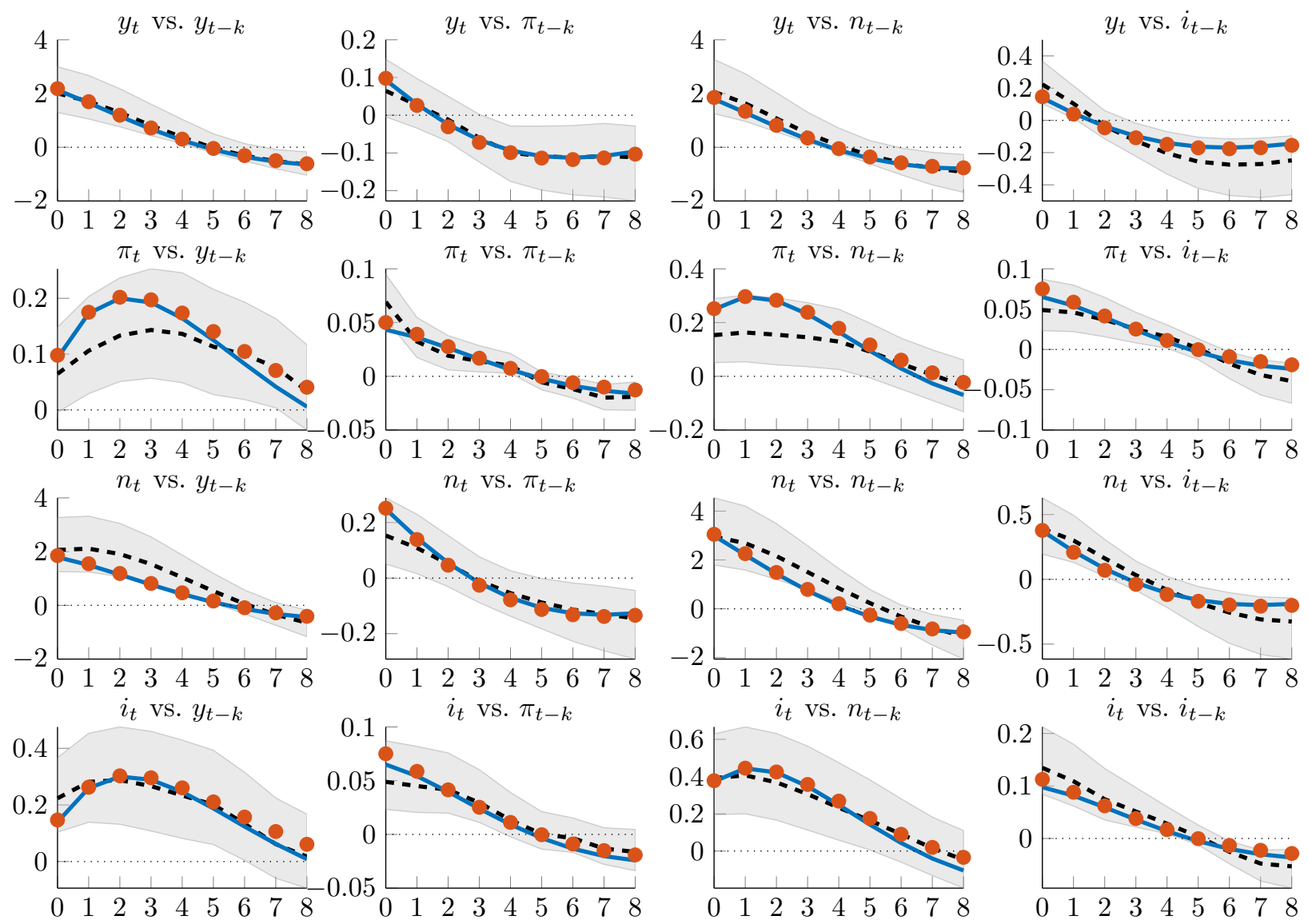

Figure 4: Business cycle comovements in the data and predicted by the estimated model. Note. - All covariances are multiplied by 100 to improve readability. Dashed black lines show the empirical covariance structure $\tilde{\Omega}_{T}$ together with 90 percent confidence intervals depicted by the shaded areas. Solid blue lines show the corresponding model moments for the $\operatorname{VAR}(1)$ case, $\tilde{\Omega}\left(\hat{\gamma}_{\text {ar }}\right)$. Red dots show the model moments for the truncated MA(14) case, $\tilde{\Omega}\left(\hat{\gamma}_{\mathrm{ma}}\right)$. Each row $i$ and column $j$ in the table shows the covariances between $\tilde{q}_{t}^{i}$ and $\tilde{q}_{t-k}^{j}$ with lags $k \in\{0,1, \ldots, 8\}$ depicted on the x-axis.

\section{D.2 Fit}

Figure 4 compares the predicted model moments with the targeted data moments. The dashed black lines show the empirical covariance structure $\tilde{\Omega}_{T}$ along with 90-percent confidence intervals (depicted by the shaded areas). The solid blue and red lines show the corresponding moments for the estimated model for the VAR(1) and MA(14) truncation of the wedges, respectively. Each row $i$ and column $j$ in the table of plots shows the covariances between $\tilde{q}_{t}^{i}$ and $\tilde{q}_{t-k}^{j}$ with lags $k \in\{0,1, \ldots, 8\}$ depicted on the horizontal axis. Despite the parametric restriction on $\tau_{t}$ and $a_{t}$ and the fact that we have less shocks than data series, the unrestricted-wedge model does a very good job at capturing the auto-covariance structure of the four time series. In addition, there is no notable difference between the $\operatorname{VAR}(1)$ and MA(14) truncation of $\tau_{t}$. 


\section{D.3 Proof of Lemma 7}

Let

$$
J=\left(\tilde{\Omega}_{T}-\tilde{\Omega}(\gamma)\right)^{\prime} W^{-1}\left(\tilde{\Omega}_{T}-\tilde{\Omega}(\gamma)\right)
$$

denote the penalty function in terms of BK-filtered moments, where the filter is applied to both the data and the model. In this appendix, we demonstrate how the penalty can be expressed in terms of the variance over unfiltered first-differenced moments, $\Omega \equiv$ vech $\left\{\operatorname{Var}\left(\mathrm{d} q_{t-K}^{t}\right)\right\}$, where $\mathrm{d}$ is the first-difference operator, and $K \equiv k+2 \bar{\tau}$ with $\bar{\tau}$ denoting the approximation horizon of the BK-filter. ${ }^{31}$ Specifically, for any positive-semidefinite $W$ we show that $J$ in (72) is equivalent to

$$
J=\left(\Omega_{T}-\Omega(\gamma)\right)^{\prime} \tilde{W}^{-1}\left(\Omega_{T}-\Omega(\gamma)\right)
$$

with $\tilde{W} \equiv\left(\Xi^{\prime} W^{-1} \Xi\right)^{-1}$ replacing $W$ (a closed-form expression for $\Xi$ is given below).

The Baxter and King (1999) filtered version of $s_{t}$ takes the form

$$
\tilde{q}_{t}=\sum_{j=-\bar{\tau}}^{\bar{\tau}} a_{j} q_{t-j}
$$

where $\tilde{q}_{t}$ is stationary by construction. For the high-pass filter used in this paper, the weights $\left\{a_{j}\right\}$ are given by

$$
a_{j}=\tilde{a}_{j}-\frac{1}{2 \bar{\tau}+1} \sum_{j=-\bar{\tau}}^{\bar{\tau}} \tilde{a}_{j}
$$

with

$$
\tilde{a}_{0}=1-\bar{\omega} / \pi, \quad \tilde{\alpha}_{j \neq 0}=-\sin (j \bar{\omega}) /(j \pi), \quad \bar{\omega}=2 \pi / 32 .
$$

To construct the filter-matrix $\Xi$, rewrite $\tilde{q}_{t}$ in terms of growth rates to get

$$
\tilde{q}_{t}=\sum_{j=-\bar{\tau}}^{\bar{\tau}} \sum_{l=0}^{\infty} a_{j} \mathrm{~d} q_{t-j-l} .
$$

Noting that $\sum_{j=-\bar{\tau}}^{\bar{\tau}} a_{j}=0$, we can simplify to get

$$
\tilde{q}_{t}=B \mathrm{~d} q_{t-\bar{\tau}-j}^{t+\bar{\tau}}
$$

\footnotetext{
${ }^{31}$ The first-difference filter is applied to the unfiltered variables to ensure stationarity for variables that have a unit root. Our transformation includes an adjustment term that corrects for the fact that the filtered moments in $\tilde{\Omega}$ are about levels rather than first-differences.
} 
where

$$
B=\left[b_{-\bar{\tau}}, \ldots, b_{\bar{\tau}}\right] \otimes I_{n},
$$

$n=4$ is the number of variables in $\tilde{q}_{t}$, and $b_{s}=\sum_{j=-\bar{\tau}}^{s} \alpha_{j}$.

Letting $L_{j}$ define the backshift matrix

$$
L_{j}=\left[\begin{array}{lll}
0_{n(2 \bar{\tau}+1), n j}, & I_{n(2 \bar{\tau}+1)}, \quad 0_{n(2 \bar{\tau}+1), n(k-j)}
\end{array}\right],
$$

we then have that

$$
\tilde{\Sigma}_{j} \equiv \operatorname{Cov}\left(\tilde{q}, \tilde{q}_{t-j}\right)=B L_{0} \Sigma^{K} L_{j}^{\prime} B^{\prime}
$$

or, equivalently,

$$
\operatorname{vec}\left(\tilde{\Sigma}_{j}\right)=\left(B L_{j} \otimes B L_{0}\right) \operatorname{vec}\left(\Sigma^{K}\right) .
$$

To complete the construction of $\Xi$, define selector-matrices $P_{0}$ and $P_{1}$ such that

$$
\operatorname{vech}\left(\tilde{\Sigma}^{k}\right)=P_{0}\left[\begin{array}{c}
\operatorname{vec}\left(\tilde{\Sigma}_{0}\right) \\
\vdots \\
\operatorname{vec}\left(\tilde{\Sigma}_{k}\right)
\end{array}\right]
$$

and

$$
\operatorname{vec}\left(\Sigma^{K}\right)=P_{1} \operatorname{vech}\left(\Sigma^{K}\right) .
$$

Stacking up $\operatorname{vec}\left(\tilde{\Sigma}_{j}\right)$, we then get

$$
\tilde{\Omega}=\Xi \Omega
$$

where

$$
\Xi=P_{0}\left[\begin{array}{c}
B L_{0} \otimes B L_{0} \\
\vdots \\
B L_{k} \otimes B L_{0}
\end{array}\right] P_{1}
$$

with $B$ and $L_{j}$ as in (74) and (75). Substitution in (72) yields (73).

\section{E Market Clearing in the Primal Economy}

There are three sets of competitive markets operating at each date $t$ in our model: a continuum of labor markets $i \in[0,1]$, an aggregate market for output, and the bond market. Local labor market clearing is ensured by full information regarding $W_{i, t}$. As usual, market clearing for bonds is implied by households' budget constraints in conjunction with good 
market clearing. Imposing goods market clearing in the primal economy yields

$$
\mathbb{E}_{t}\left[r_{t}+\mathrm{d} \tau_{t+1}^{c}\right]=\mathbb{E}_{t}\left[\mathrm{~d} \hat{y}_{t+1}\right]=\xi \mathbb{E}_{t}\left[\mathrm{~d} \tau_{t+1}^{x}\right]
$$

For markets to clear, the real interest rate $r_{t}=\phi \pi_{t}-\mathbb{E}_{t} \pi_{t+1}$ has to adjust so that consumers' demand - taking into account households' errors in their consumption decisions $\tau_{t}^{c}$-matches the output gap as determined by $\tau_{t}^{x}$. Clearly, for any stationary process for $\tau_{t}^{c}$ and $\tau_{t}^{x}$, there exists a process for $r_{t}$ so that (77) holds.

The key insight is that in the primal economy, any error made by households in their consumption choice as reflected in $\mathbb{E}_{t}\left[\mathrm{~d} \tau_{t+1}^{c}\right]$ is endogenously offset by fluctuations in the real interest rate. Intuitively, the degree of "awareness" regarding variations in the real interest rate endogenously adjusts so that prices can perform their market clearing role. This is fundamentally different from a parametric structure in which the expectation error is determined endogenously and there may not be any solution to (77).

As an example, suppose consumers are perfectly informed about future consumption, so we can interpret $\mathbb{E}_{t}\left[r_{t}+\mathrm{d} \tau_{t+1}^{c}\right]$ as the average expected interest rate, $\overline{\mathbb{E}}_{t}\left[r_{t}\right]$. With a parametric information structure, we would need to ensure that $\overline{\mathbb{E}}_{t}\left[r_{t}\right]$ is sufficiently responsive to the output gap, $\xi \tau_{t}^{x}$, which fails, e.g., if consumers have no information regarding $r_{t}$. By contrast, the primal approach endogenously pins down $\overline{\mathbb{E}}_{t}\left[r_{t}\right]$ as the market-clearing object and fixing $\tau_{t}^{c}$ merely determines how $r_{t}$ fluctuates with $\tau_{t}^{x}$ so that $r_{t}+\mathbb{E}_{t}\left[\mathrm{~d} \tau_{t+1}\right]$ clears the market. 


\section{References}

Acharya, Sushant. 2013. "Dispersed Beliefs and Aggregate Demand Management." working paper.

Acharya, Sushant, Jess Benhabib, and Zhen Huo. 2017. "The Anatomy of SentimentDriven Fluctuations." National Bureau of Economic Research, Cambridge, MA.

Amador, Manuel, and Pierre-Olivier Weill. 2010. "Learning from prices: Public communication and welfare." Journal of Political Economy, 118(5): 866-907.

Angeletos, George-Marios, and Jennifer La'O. 2010. "Noisy Business Cycles." NBER Macroeconomics Annual, 24.

Angeletos, George-Marios, and Jennifer La'O. 2013. "Sentiments." Econometrica, 81(2): 739-779.

Angeletos, George-Marios, Fabrice Collard, and Harris Dellas. 2017. "Quantifying Confidence." working paper.

Angeletos, George-Marios, Fabrice Collard, and Harris Dellas. 2018. "Business Cycle Anatomy." National Bureau of Economic Research Working Paper 24875.

Baxter, Marianne, and Robert G. King. 1999. "Measuring business cycles: Approximate band-pass filters for economic time series." Review of Economics and Statistics, 81(4): 575-593.

Benhabib, Jess, Pengfei Wang, and Yi Wen. 2015. "Sentiments and Aggregate Demand Fluctuations." Econometrica, 83(2): 549-585.

Bergemann, Dirk, and Stephen Morris. 2013. "Robust Predictions in Games With Incomplete Information." Econometrica, 81(4): 1251-1308.

Bergemann, Dirk, and Stephen Morris. 2016. "Bayes correlated equilibrium and the comparison of information structures in games." Theoretical Economics, 11(2): 487-522.

Bergemann, Dirk, Tibor Heumann, and Stephen Morris. 2014. "Information and Volatility." mimeo.

Blanchard, Olivier J, Jean-Paul L'Huillier, and Guido Lorenzoni. 2013. "News, Noise, and Fluctuations: An Empirical Exploration." American Economic Review, 103(7): 3045-3070. 
Chahrour, Ryan, and Gaetano Gaballo. 2016. "Learning from Prices: Amplification and Business Fluctuations." working paper.

Chahrour, Ryan, and Kyle Jurado. 2017. "Recoverability." Economic Research Initiatives at Duke (ERID) Working Paper, , (259).

Chahrour, Ryan, and Robert Ulbricht. 2017. "Information-driven Business Cycles: A Primal Approach." TSE Working Paper, n. 17-784.

Chari, V. V., Patrick J. Kehoe, and Ellen R. McGrattan. 2007. "Business cycle accounting." Econometrica, 75(3): 781-836.

Cochrane, John H. 1994. "Shocks." Vol. 41, 295-364, Elsevier.

Coibion, Olivier, and Yuriy Gorodnichenko. 2014. "Information Rigidity and the Expectations Formation Process: A Simple Framework and New Facts." American Economic Review, forthcoming.

Foster, Lucia, John Haltiwanger, and Chad Syverson. 2008. "Reallocation, Firm Turnover, and Efficiency: Selection on Productivity or Profitability?" American Economic Review, 98(1): 394-425.

Gorodnichenko, Yuriy, and Serena Ng. 2009. "Estimation of DSGE Models when the Data are Persistent." working paper.

Hansen, Lars Peter, and Thomas J. Sargent. 1980. "Formulating and estimating dynamic linear rational expectations models." Journal of Economic Dynamics and Control, 2: $7-46$.

Hansen, Lars Peter, and Thomas J. Sargent. 1981. "A note on Wiener-Kolmogorov prediction formulas for rational expectations models." Economics Letters, 8(3): 255-260.

Hellwig, Christian, and Venky Venkateswaran. 2014. "Dispersed Information, Sticky Prices and Monetary Business Cycles: A Hayekian Perspective." working paper.

Huo, Zhen, and Naoki Takayama. 2015a. "Higher Order Beliefs, Confidence, and Business Cycles." working paper.

Huo, Zhen, and Naoki Takayama. 2015b. "Rational Expectations Models with Higher Order Beliefs." working paper. 
Ilut, Cosmin, and Hikaru Saijo. 2018. "Learning, Confidence, and Business Cycles." working paper.

Lorenzoni, Guido. 2009. "A Theory of Demand Shocks." The American Economic Review, 99(5): 2050-2084.

Lucas, Robert E. 1972. "Expectations and the Neutrality of Money." Journal of Economic Theory, 124: 103-124.

Lucas, Robert E. 1973. "Some international evidence on output-inflation tradeoffs." The American Economic Review, 63(3): 326-334.

Maćkowiak, Bartosz, and Mirko Wiederholt. 2009. "Optimal Sticky Prices under Rational Inattention." The American Economic Review, 99(3): 769-803.

Maćkowiak, Bartosz, and Mirko Wiederholt. 2015. "Business cycle dynamics under rational inattention." Review of Economic Studies, 82(4): 1502-1532.

Melosi, Leonardo. 2014. "Estimating models with dispersed information." American Economic Journal: Macroeconomics, 6(1 C): 1-31.

Melosi, Leonardo. 2016. "Signaling Effects of Monetary Policy." Review of Economic Studies, forthcoming.

Nimark, Kristoffer P. 2009. "Dynamic higher order expectations." mimeo.

Passadore, Juan, and Juan Pablo Xandri. 2018. "Robust Predictions in Dynamic Policy Games." working paper.

Rondina, Giacomo, and Todd B Walker. 2014. "Dispersed Information and Confounding Dynamics." mimeo.

Sims, Christopher A. 2003. "Implications of rational inattention." Journal of Monetary Economics, 50(3): 665-690.

Vives, Xavier, and Liyan Yang. 2017. "Costly Interpretation of Asset Prices." working paper.

Whiteman, Charles. 1983. Linear Rational Expectations Models: A User's Guide. University of Minnesota Press. 
Woodford, Michael. 2003. "Imperfect common knowledge and the effects of monetary policy." In Knowledge, Information and Expectations in Modern Macroeconomics: In Honor of Edmund S. Phelps. , ed. Philippe Aghion, Roman Frydman, Joseph Stiglitz and Michael Woodford, 25-58. Princeton:Princeton University Press. 